University of Nebraska - Lincoln

DigitalCommons@University of Nebraska - Lincoln

Publications, Agencies and Staff of the U.S.

Department of Commerce

U.S. Department of Commerce

2011

Measuring information content from observations for data assimilations: utilities of spectral formulations demonstrated with radar observations

Qin Xu

NOAA/National Severe Storms Laboratory, qin.xu@noaa.gov

Li Wei

University of Oklahoma Norman Campus

Follow this and additional works at: https://digitalcommons.unl.edu/usdeptcommercepub

Part of the Environmental Sciences Commons

$\mathrm{Xu}$, Qin and Wei, Li, "Measuring information content from observations for data assimilations: utilities of spectral formulations demonstrated with radar observations" (2011). Publications, Agencies and Staff of the U.S. Department of Commerce. 334.

https://digitalcommons.unl.edu/usdeptcommercepub/334

This Article is brought to you for free and open access by the U.S. Department of Commerce at DigitalCommons@University of Nebraska - Lincoln. It has been accepted for inclusion in Publications, Agencies and Staff of the U.S. Department of Commerce by an authorized administrator of DigitalCommons@University of Nebraska - Lincoln. 


\title{
Measuring information content from observations for data assimilations: utilities of spectral formulations demonstrated with radar observations
}

\author{
By Q IN X U ${ }^{1 *}$ and LI W EI ${ }^{2}, \quad{ }^{1}$ NOAA/National Severe Storms Laboratory, Norman, Oklahoma, USA; \\ ${ }^{2}$ Cooperative Institute for Mesoscale Meteorological Studies, University of Oklahoma, Norman, Oklahoma, USA
}

(Manuscript received 22 May 2011; in final form 14 July 2011)

\begin{abstract}
Utilities of the spectral formulations for measuring information content from observations are explored and demonstrated with real radar data. It is shown that the spectral formulations can be used (i) to precisely compute the information contents from one-dimensional radar data uniformly distributed along the radar beam, (ii) to approximately estimate the information contents from two-dimensional radar observations non-uniformly distributed on the conical surface of radar scan and thus (iii) to estimate the information losses caused by super-observations generated by local averaging with a series of successively coarsened resolutions to find an optimally coarsened resolution for radar data compression with zero or near-zero minimal loss of information. The results obtained from the spectral formulations are verified against the results computed accurately but costly from the singular-value formulations. As the background and observation error power spectra can be derived analytically for the above utilities, the spectral formulations are computationally much more efficient and affordable than the singular-value formulations, even and especially when the background space and observation space are both extremely large and too large to be computed by the singular-value formulations.
\end{abstract}

\section{Introduction}

Remotely sensed observations, especially those scanned from radars, are often much denser than the model background and analysis grids used in data assimilations for numerical weather predictions. Excessive observation resolutions not only impose unnecessary computational burdens on a data analysis system but can also cause the analysis system to be ill conditioned. To reduce or eliminate observation resolution redundancy for data assimilation, it is necessary to compress densely distributed observations into fewer super-observations with minimum possible loss of information. Such observational data compression is called super-Obbing. Some general super-Obbing techniques were proposed by Purser et al. (2000) to minimize the information loss measured by the Shannon entropy difference with the observations batched locally around each super-observation. With these techniques, super-observations can be generated efficiently by weighted local averaging, but the information loss is minimized locally rather than globally. By converting the general formulations of relative entropy and Shannon entropy difference into

*Corresponding author: National Severe Storms Laboratory, 120 David L. Boren Blvd., Norman, Oklahoma 73072-7326, USA. e-mail: Qin.Xu@noaa.gov

DOI: 10.1111/j.1600-0870.2011.00542.x singular-value forms for measuring information content from observations, $\mathrm{Xu}$ (2007, referred to as X07 hereafter) examined the above issue without batching the observations locally. With the singular-value formulations, the information redundancy from densely distributed observations can be explicitly quantified in connection with the rank deficiency of the scaled observation operator, $\mathbf{M} \equiv \mathbf{R}^{-1 / 2} \mathbf{H B}^{1 / 2}$, and super-observations can be constructed with zero or minimized information loss globally. However, since the super-observations and their covariance are constructed in the truncated singular-vector space of $\mathbf{M}$, their connections to the original observations are non-local and non-explicit in the physical space, and the singular-value decomposition (SVD) of $\mathbf{M}$ will become too expensive or impractical to compute if the background space and observation space are both very large, as often seen in radar data assimilation (Gao et al., 2004; Xu et al., 2010).

To overcome the above shortcomings, $\mathrm{Xu}$ (2011, referred to as X11 hereafter) transformed the singular-value formulations into spectral forms in the wavenumber space for situations in which the observations are locally uniform and the observation and background error covariances or correlations (i.e. the covariances scaled by their respective variances) are locally homogeneous in each direction in the analysis domain. The spectral formulations exhibited the following advantages over their counterpart singular-value formulations: (i) The information contents 
from densely distributed observations can be calculated very efficiently and analysed easily for each wavenumber. (ii) Superobservations can be constructed by spectral truncations in the wavenumber space with zero or minimum loss of information and explicitly related to the original observations in the physical space. These advantages, however, have not been tested and demonstrated yet with real observations. As a follow-up of X11, this paper will design and perform these tests with radar observations and explore the utilities of the spectral formulations for radar data compression.

As shown and commented in section 4.1 of X11, constructing super-observations by a spectral truncation requires the observations to be precisely uniformly distributed, and this is a serious limitation for super-Obbing by spectral truncation. On the other hand, super-Obbing by local averaging or weighted local averaging can be used easily and adaptively for any densely distributed observations without the above limitation (see e.g. Purser et al., 2000; Liu et al., 2005; Alpert and Kumar 2007; Lu et al. 2011, section 3.3). According to the theoretical analysis based on the spectral formulations in section 4.3 and remarks in section 4.4 of X11, super-Obbing by local averaging will cause no loss of information (measured globally by the dispersion part of relative entropy or the Shannon entropy difference) for uniformly distributed observations with unbiased and spatially uncorrelated errors unless the super-observation resolution becomes coarser than the effective background resolution (Frehlich, 2008) or the background error covariance resolution (determined by one half of the wavelength associated with the cut-off wavenumber of the background error power spectrum). This is an attractive global property for super-Obbing by local averaging, but it is not clear whether and to what extent this global property can be retained for non-uniformly distributed observations. It is also not clear whether and how the information loss (if any) caused by local averaging for non-uniformly distributed observations can be estimated by the spectral formulations. These problems will be examined in this paper. In particular, we will explore how to apply the spectral formulations to densely but non-uniformly distributed radar observations as well as to their compressed super-observations by local averaging, so the information loss (if any) caused by local averaging can be estimated efficiently as a function of the successively coarsened super-observation resolution to find an optimally coarsened resolution for superObbing by local averaging that causes zero or near-zero minimal loss of information.

The paper is organized as follows. The next section will examine the utility and accuracy of the spectral formulations, in comparison with the singular-value formulations, for computing the information contents from one-dimensional radar observations uniformly distributed along the radar beam and the information losses caused by spectral truncation, uniform thinning and local averaging. Section 3 will explore and demonstrate the utility of the spectral formulations, in comparison with the singular-value formulations, for estimating the information contents from two- dimensional radar observations non-uniformly distributed on the conical surface of radar scan and the information losses caused by local averaging with a series of successively coarsened resolutions. Conclusions follow in Section 4.

\section{Applications to one-dimensional radar data along the radar beam}

\subsection{Descriptions of the one-dimensional radar data and background field}

The selected radar data are the same as those described in section 5.2 of X07, and they are the radial-component velocities (along the radar beam) scanned by the NSSL phased array radar from 2100 to 2200 UTC on 2 June 2004 with a resolution of $240 \mathrm{~m}$ in the radial direction and a resolution of $1.6^{\circ}$ in the azimuthal direction. The data were processed through quality control and then thinned to the coarsened resolution of $\Delta x_{o}=3 \mathrm{~km}$ along the radar beam (see fig. 1 of X07). After the thinning, the observation errors become spatially uncorrelated (Xu et al., 2007) to facilitate the tests in Section 2.5 for super-Obbing by local averaging to cause zero or minimal information loss. The observation error variance is $\sigma_{0}^{2}=6.4 \mathrm{~m}^{2} \mathrm{~s}^{-2}$, as estimated in $\mathrm{Xu}$ et al. (2005). The single beam of radial-velocity observations that was used for the illustrative examples in section 5 of $\mathrm{X} 07$ is used here again. This beam was scanned at 2108 UTC along $0.75^{\circ}$ elevation angle and $97.8^{\circ}$ azimuthal angle (positive clockwise from the north), and it contains 40 (thinned) observations (spaced every $\Delta x_{o}=$ $3 \mathrm{~km}$ ). The observation error covariance matrix is thus given by $\mathbf{R}=\sigma_{0}^{2} \mathbf{I}_{M}$ with $M=40$.

The background velocity field is produced by the Coupled Ocean/Atmosphere Mesoscale Prediction System (COAMPS, Hodur, 1997) on a $6 \mathrm{~km}$ grid and then projected onto the aforementioned radar beam on a one-dimensional grid of $\Delta x=6 \mathrm{~km}$ resolution with $N=20$, where $N$ denotes the number of grid points. This one-dimensional grid is similar to that described in section 5.2 of X07, except that the resolution is $6 \mathrm{~km}$ instead of $5 \mathrm{~km}$ and thus the number of grid points is 20 instead of 24 . In this case, the background radial-velocity error covariance still can be modelled approximately by the Gaussian function, that is, $\sigma^{2} \exp \left[-x^{2} /\left(2 L^{2}\right)\right]$ according to (2.7) and (3.2) of $\mathrm{Xu}$ and Gong (2003), where $x$ is the distance between two correlated points along the radar beam, $\sigma^{2}$ and $L$ denote the background radial-velocity error variance and de-correlation length scale, respectively. As in section 5.4 of X07, $\sigma^{2}=70 \mathrm{~m}^{2} \mathrm{~s}^{-2}$ and $L=$ $15 \mathrm{~km}$ are selected here for the illustrative purpose. However, to use the spectral formation, the above one-dimensional domain of length $D=M \Delta x_{o}=N \Delta x(=120 \mathrm{~km})$ needs to be extended periodically as described in section 2.2 of X11. In particular, the background error covariance function can be extended periodically into

$$
B(x)=\sigma^{2} \exp \left\{-(|x|-\operatorname{Int}[|x| / D+1 / 2] D)^{2} /\left(2 L^{2}\right)\right\},
$$


where Int[ ] denotes the integer part of [ ]. This periodic covariance function recovers exactly $B(x)=\sigma^{2} \exp \left[-x^{2} /\left(2 L^{2}\right)\right]$ for $|x| \leq D / 2$ in the original domain, but it is continuous only to the zeroth order and thus non-smooth at $|x|=(1 / 2+i) D$ for $i=0$, $1,2, \ldots$ For $D \gg L$, the background error covariance function can be also extended periodically into

$B(x)=\left(\sigma^{2} / A_{0}\right) \sum_{i=-\infty}^{\infty} \exp \left[-(x-i D)^{2} /\left(2 L^{2}\right)\right]$,

where $A_{0}=\sum_{i=-\infty}^{\infty} \exp \left[-(i D)^{2} /\left(2 L^{2}\right)\right]$ to ensure $B(0)=\sigma^{2}$. For the above selected $D=120 \mathrm{~km}$ and $L=15 \mathrm{~km}, A_{0}=1+$ $2.5 \times 10^{-14} \approx 1$ and thus $(1 \mathrm{~b})$ recovers $B(x)=\sigma^{2} \exp \left[-x^{2} /\left(2 L^{2}\right)\right]$ almost exactly for $|x| \leq D / 2$ in the original domain. Since the periodic covariance function in (1b) is smooth to infinitely high order and its associated power spectrum has a simple analytical form [see (10)], (1b) will be used in this section as the periodically extended covariance function. The background error covariance matrix $\mathbf{B}$ can be constructed from $B(x)$ in (1b) with the $n$th element in the $n^{\prime}$ th column of $\mathbf{B}$ given by $B\left(n \Delta x-n^{\prime} \Delta x\right)$ for $n$ and $n^{\prime}$ in the range of $\left[N_{-}, N_{+}\right]$, where $N_{-} \equiv \operatorname{Int}[(1-$ $N) / 2]=-19$ and $N_{+} \equiv \operatorname{Int}[N / 2]=20$ for $N=40$.

\subsection{Applications of spectral formulations versus singular-value formulations}

According to (8) of Xu et al. (2009) and (22)-(24) of X11, the spectral formations for the signal part of the relative entropy (denoted by $S g$ ), the dispersion part of the relative entropy (denoted by $D s$ ), the Shannon entropy difference (denoted by $S D$ ), and the degrees of freedom for signal (denoted by DFS), can be written into the following forms:

$S g=\sum_{i=\mu_{-}}^{\mu_{+}} S g_{i}$

$D s=\sum_{i=\mu_{-}}^{\mu_{+}} D s_{i}$,

$S D=\sum_{i=\mu_{-}}^{\mu_{+}} S D_{i}$,

$D F S=\sum_{i=\mu_{-}}^{\mu_{+}} D F S_{i}$

Here, $\mu_{-} \equiv \operatorname{Int}[(1-\mu) / 2], \mu_{+} \equiv \operatorname{Int}[\mu / 2]$ and $\mu \equiv$ $\min (N, M)$;

$$
\begin{aligned}
S g_{i} & =\left[\left(\left|c_{i}\right|^{2} / C_{i}\right)\left|\gamma_{i}\right|^{2} /\left(1+\left|\gamma_{i}\right|^{2}\right)^{2}\right] / 2 \\
& =\left[\left|c_{i}\right|^{2} /\left(2 \beta \Pi_{i}\right)\right]\left|\gamma_{i}\right|^{4} /\left(1+\left|\gamma_{i}\right|^{2}\right)^{2}, \\
D s_{i} & =S D_{i}-D F S_{i} / 2,
\end{aligned}
$$

$S D_{i}=\ln \left(1+\left|\gamma_{i}\right|^{2}\right) / 2$

and

$D F S_{i}=\left|\gamma_{i}\right|^{2}\left(1+\left|\gamma_{i}\right|^{2}\right)$,

are the $i$ th components of $S g, D s, S D$ and $D F S$, respectively, in the spectral space; and $c_{i}$ is the $i$ th component of the normalized discrete Fourier transform (DFT) of the innovation $d_{m}$ [see (10)-(11) of X11]; $C_{i}$ is the observation error power spectrum [see (14) of X11]; $\Pi_{i}$ is the collapsed background error power spectrum (into the range of the observation error spectrum) if $N>M$ but becomes identical to the background error power spectrum, denoted by $S_{i}$, if $N \leq M$ [see (13) and (25) of X11]; $\left|\gamma_{i}\right|^{2}=\beta \Pi_{i} / C_{i}$ and $\beta=\Delta x / \Delta x_{o}$.

For the data described in Section 2.1, the number of observations $(M=40)$ is larger than the number of the background grid points $(N=20)$ and the observation errors are spatially uncorrelated, so $\Pi_{i}=S_{i}, C_{i}=\sigma_{0}^{2}$ and $\left|\gamma_{i}\right|^{2}=\beta S_{i} / \sigma_{0}^{2}$. By using the fast Fourier transform (FFT), $S_{i}$ and $c_{i}$ can be computed efficiently. For $B(x)$ given in (1b), $S_{i}$ can also be obtained analytically by using the following generalized Fourier Transformation:

$$
\begin{aligned}
S(k) & =\int_{-\infty}^{\infty} d x B(x) \exp (-j k x) \\
& =\left(\sigma^{2} / A_{0}\right) L(2 \pi)^{1 / 2} \exp \left(-k^{2} L^{2} / 2\right) \sum_{-\infty}^{\infty} \exp (-j i k D), \\
& =\left(\sigma^{2} / A_{0}\right) L(2 \pi)^{1 / 2} \exp \left(-k^{2} L^{2} / 2\right) \sum_{i=-\infty}^{\infty} \delta\left(k-k_{i}\right),
\end{aligned}
$$

where $j \equiv(-1)^{1 / 2}$ is the imaginary unit, $k$ is the wavenumber, $\delta(\bullet)$ denotes the delta function of $(\bullet)$, $\int_{-\infty}^{\infty} d x \exp \left[-(x-i D)^{2} /\left(2 L^{2}\right)\right]=L(2 \pi)^{1 / 2} \exp \left(-k^{2} L^{2} / 2\right)$ $\exp (-j i k D)$ is used in the second step, and the identity $\sum_{i=-\infty}^{\infty} \exp (-j i k D)=(2 \pi / D) \sum_{i=-\infty}^{\infty} \delta(k-2 \pi i / D) \quad$ is used with $k_{i}=i \Delta k$ and $\Delta k \equiv 2 \pi / D$ in the third step (see page 68 of Lighthill, 1958). Note that the Fourier integral that defines $S(k)$ in (10) can be discretized into $\sum_{n=N_{-}}^{N_{+}} B\left(x_{n}\right) \exp \left(-j k_{i} x_{n}\right) \Delta x=S_{i} \Delta x$ over the periodic domain $D$, where $S_{i}=S\left(k_{i}\right)$ is precisely the same discrete power spectrum as that defined in (12)-(13) of X11. On the other hand, the analytical form of $S(k)$ derived in (10) can be discretized directly into $\int_{(i-1 / 2) \Delta k}^{(i+1 / 2) \Delta k} d k S(k)$. According to (10), the above two discretized forms should be equal to each other, and this leads to the following analytical expression of $S_{i}$

$$
\begin{aligned}
S_{i} & =\int_{(i-1 / 2) \Delta k}^{(i+1 / 2) \Delta k} d k S(k) / \Delta x \\
& =\left(\sigma^{2} / A_{0}\right)(L / \Delta x)(2 \pi)^{1 / 2} \exp \left(-k_{i}^{2} L^{2} / 2\right) .
\end{aligned}
$$

As shown above, $S_{i}$ can be obtained either numerically by using the DFT in (12) of X11 [which is equivalent to the discrete cosine transform in (13) of in X11] or analytically by using (11). For the data described in Section 2.1, these two ways give almost identical $S_{i}$ and thus almost identical $\left|\gamma_{i}\right|=\left(\beta S_{i}\right)^{1 / 2} / \sigma_{o}$. For $|i| \leq$ 
$6,\left|\gamma_{i}\right|$ is obtained identically at least to the 5 th effective digit with the exact symmetry of $\left|\gamma_{-i}\right|=\left|\gamma_{i}\right|$, and the obtained values are $\left|\gamma_{0}\right|=11.848,\left|\gamma_{1}\right|=10.155,\left|\gamma_{2}\right|=6.3936,\left|\gamma_{3}\right|=2.9572$, $\left|\gamma_{4}\right|=1.0048,\left|\gamma_{5}\right|=0.25078$ and $\left|\gamma_{6}\right|=0.045982$. For $|i| \geq$ $7,\left|\gamma_{i}\right|$ is also obtained with the exact symmetry of $\left|\gamma_{-i}\right|=$ $\left|\gamma_{i}\right|$, but the values obtained by the two ways become slightly different, and the numerically (or analytical) obtained values are $\left|\gamma_{7}\right|=6.1935 \times 10^{-3}$ (or $\left.6.1934 \times 10^{-3}\right),\left|\gamma_{8}\right|=6.1191 \times 10^{-4}$ (or $\left.6.1281 \times 10^{-4}\right),\left|\gamma_{9}\right|=5.1068 \times 10^{-5}$ (or $\left.4.4542 \times 10^{-5}\right)$, and $\left|\gamma_{10}\right|=3.3613 \times 10^{-6}$ (or $\left.2.3783 \times 10^{-6}\right)$. Here, since $M=$ 40 and $N=20$, the wavenumber index $i$ ranges from $\mu_{-}=-9$ to $\mu_{+}=10$ in (2)-(5), and the index range length is $\mu=\mu_{+}-$ $\mu_{-}+1=20$. Due to the symmetry of $\left|\gamma_{-i}\right|=\left|\gamma_{i}\right|$, there are only 11 independent values of $\left|\gamma_{i}\right|$ as listed above for $0 \leq i \leq$ 10.

In the singular-value formulations [see (3.6)-(3.10) of X07], the singular-value index $i$ ranges from 1 to $\mu$, so the index range length is also $\mu=20$. Since $\left|\gamma_{i}\right|=\left(\beta S_{i}\right)^{1 / 2} / \sigma_{o}$ is a decreasing function of $|i|$ as shown in (11), $\left|\gamma_{i}\right|$ for $i>0$ (or $i \leq 0$ ) in the spectral formulations is equivalent to $\lambda_{2 i}$ (or $\lambda_{2 i+1}$ ) - the (2i)th [or $(1+2 i)$ th] singular value in the singular-value formulations. This equivalence was explained in the conclusion of X11 and can be verified here numerically by comparing the above obtained $\left|\gamma_{i}\right|$ for $i>0$ (or $i \leq 0$ ) with $\lambda_{2 i}$ (or $\lambda_{2 i+1}$ ) computed from the SVD of the scaled observation operator $\mathbf{M} \equiv \mathbf{R}^{-1 / 2} \mathbf{H B} \mathbf{B}^{1 / 2}$ [see (3.6) of X07]. The computed $\lambda_{1}, \lambda_{2}, \lambda_{3}, \ldots \lambda_{19}$ are found to be identical (at least to the 5 th effective digit) to the above analytically derived $\left|\gamma_{0}\right|,\left|\gamma_{1}\right|,\left|\gamma_{-1}\right|, \ldots\left|\gamma_{-9}\right|$, respectively. Only the last and smallest singular value $\lambda_{20}\left(=3.3635 \times 10^{-6}\right)$ becomes different from the above obtained $\left|\gamma_{10}\right|$ mainly due to computer round-off errors. This well-verified equivalence is also shown in Fig. 1 by the two coincided curves, that is, (i) the grey dashed curve labelled 'SVD-Svi' for the SVD-computed $\lambda_{i} / \lambda_{1}$ (called the relative amplitude of $\lambda_{i}$ ), and (ii) the thin solid curve labelled 'Spc-Svi' for the spectral-derived $\left|\gamma_{i} / \gamma_{0}\right|$ (called the relative amplitude of $\left.\left|\gamma_{i}\right|\right)$ plotted in the wavenumber index sequence of $i=0,1,-1, \ldots, 7,-7$ (not shown) corresponding to the singular-value index sequence from $i=1$ to 15 (as shown explicitly). According to the above comparisons, the spectralderived $S_{i}$ in (11) is sufficiently accurate and will be used for the applications considered in this section.

With $S_{i}$ given by (11) and $c_{i}$ given by the DFT of the innovation $d_{m}$, the signal part of the relative entropy $S g$ and its partitioned component, denoted by $S g_{i}$ for the $i$ th wavenumber $k_{i}$, can be computed from (2) and (6), respectively. The computed $S g_{i} / S g$ is plotted in Fig. 1 by the dark dotted curve labelled 'Spc-Sgi' in the wavenumber index sequence of $i=0,1,-1, \ldots 7,-7$ (not shown) corresponding to the singular-value index sequence from $i=1$ to 15 (as shown explicitly). The relative amplitude $\mathrm{Sg}_{i} / \mathrm{Sg}$ is also computed by using the singular-value formulation \{see (5.4) of X07 but note that $S g_{i}$ was already normalized by $S g$ and there was a typo in the definition of $S g$, that is, $\left[\lambda_{i}^{2}(1+\right.$ $\left.\left.\lambda_{i}^{2}\right)^{-2}\right]^{-1}$ should be $\lambda_{i}^{2}\left(1+\lambda_{i}^{2}\right)^{-2}$ inside the summation for the

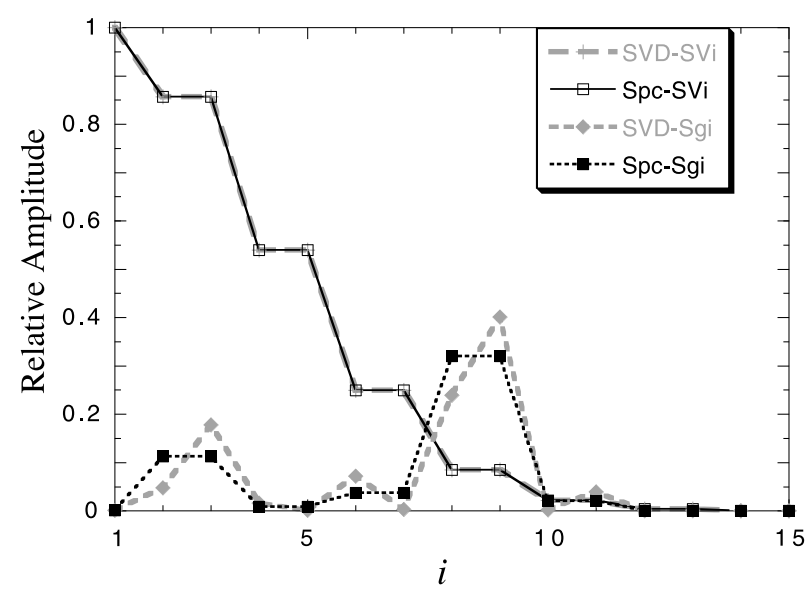

Fig. 1. Relative amplitudes of SVD-computed $\lambda_{i}$ and $S g_{i}$ plotted as functions of the singular-value index $i$ by the grey dashed curve (labelled SVD-SVi) and grey dotted curve (labelled SVD-Sgi), respectively. Relative amplitudes of Spectral-derived $\left|\gamma_{i}\right|$ and $S g_{i}$ plotted by the thin solid curve (labelled Spc-SVi) and dark dotted curve (labelled Spc-Sgi), respectively, in the wavenumber index sequence of $i=0,1,-1, \ldots, 7,-7$ (not shown) corresponding to the singular-value index sequence from $i=1$ to 15 (as shown explicitly). When the singular-value index $i$ increases beyond 15 toward its upper bound of $\mu \equiv \min (N, M)=20$ (outside the plotted range), all the relative amplitudes become extremely and increasingly close to zero (not shown). The relative amplitudes of $\lambda_{i},\left|\gamma_{i}\right|$ and $S g_{i}$ are defined by $\lambda_{i} / \lambda_{1}$, $\left|\gamma_{i} / \gamma_{0}\right|$ and $S g_{i} / S g$, respectively.

second equation in (5.4) of $\mathrm{X} 07\}$, and the result is plotted by the grey dotted curve labelled 'SVD-Sgi' as a function of the singular-value index $i$ in Fig. 1. As shown by the Spc-Sgi curve, the spectral-computed $S g_{i}$ satisfies the symmetry of $S g_{i}=S g_{-i}$ where $i$ is the wavenumber index, but the SVD-Sgi curve does not. The difference is caused by the non-uniqueness of the SVD of $\mathbf{M}$. As shown in (7)-(9) and (20)-(21) of X11, the SVD presented by $\mathbf{M}=\mathbf{U} \Lambda \mathbf{V}^{\mathrm{T}}$ in $\mathrm{X} 07$ can be generalized to a complex SVD (although $\mathbf{M}$ is always a real matrix), so the squared absolute value of the complex singular value can be given by $\left|\gamma_{i}\right|^{2}=$ $\beta S_{i} / \sigma_{o}^{2}$ for the $i$ th wavenumber $k_{i}$ and the complex counterpart of $\mathbf{U}$ can be given by the unitary matrix $F_{M}^{H}$, where $\mathbf{F}_{M}$ is the normalized DFT matrix in the observation space [see (11) of X11], and ()$^{\mathrm{H}} \equiv()^{* \mathrm{~T}},()^{*}$ and ()$^{\mathrm{T}}$ denote the Hermit transport, complex conjugate and real transpose of (), respectively. With this generalization, the transformed innovation vector $\mathbf{d}^{\prime}=\mathbf{U}^{\mathrm{T}} \mathbf{R}^{-1 / 2} \mathbf{d}$ in the singular-vector space [see (3.6) of X07] is replaced by its spectral representation $\mathbf{C}^{-1 / 2} \mathbf{c}=\mathbf{F}_{M} \mathbf{R}^{-1 / 2} \mathbf{d}$ [see (22) of X11]. Clearly, $\mathbf{F}_{M}$ is different from $\mathbf{U}^{\mathrm{T}}$, and this explains the difference between the SVD-Sgi and Spc-Sgi curves in Fig. 1. As shown by the Spc-Sgi curve, the spectral-computed $\mathrm{Sg}_{i} / \mathrm{Sg}$ and $S g_{-i} / S g$ have the same value (due to the symmetry of $S g_{i}=S g_{-i}$ ) for the $i$ th paired (positive and negative) wavenumbers and this value is the same as the averaged value of the two counterpart points 


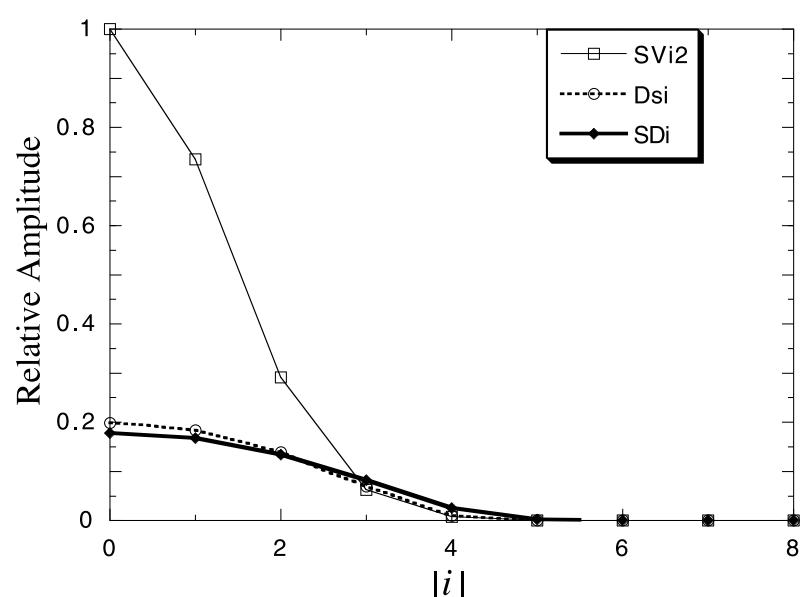

Fig. 2. Relative amplitudes of spectral-derived $\left|\gamma_{i}\right|^{2}, D s_{i}$ and $S D_{i}$ plotted by the thin solid curve (labelled SVi2), dotted curve (labelled Dsi) and thick solid curve (labelled SDi), respectively, as functions of the absolute value of the wavenumber index over the range of $0 \leq|i|<$ 8. The relative amplitudes of $\left|\gamma_{i}\right|^{2}, D s_{i}$ and $S D_{i}$ are defined by $\left|\gamma_{i} / \gamma_{0}\right|^{2}, D s_{i} / D s$ and $S D_{i} / S D$, respectively. The $S V i 2$ curve is duplicated from the square of the Spc-SVi curve in Fig. 1 but plotted here as a function of the absolute value of the wavenumber index (instead of the singular-value index).

on the SVD-Sgi curve, so the two curves yield exactly the same summed value of $S g$ (=5.994949) in (2).

The dispersion part of the relative entropy $D s$ and its partitioned component $D s_{i}$ for $k_{i}$ are formulated in (3) and (7), respectively. The Shannon entropy difference $S D$ and its partitioned component $S D_{i}$ for the $i$ th wavenumber $k_{i}$ are formulated in (4) and (8), respectively. The spectral-derived $D s_{i} / D s$ (called the relative amplitude of $D s_{i}$ ) and $S D_{i} / S D$ (called the relative amplitude of $S D_{i}$ ) are plotted in Fig. 2 by the dark dotted and dark solid curves, respectively, as functions of the absolute value of the wavenumber index over the range of $0 \leq|i|<8$. The SpcSVi curve in Fig. 1 is squared and recast into the thin solid curve labelled 'SVi2' for the spectral-derived $\left|\gamma_{i} / \gamma_{0}\right|^{2}$ as a function of $|i|$, where $i$ is the wavenumber index (instead of the singularvalue index in Fig. 1). Note from (7)-(8) that $D s_{i}$ and $S D_{i}$ are determined solely by $\left|\gamma_{i}\right|$, and $\left|\gamma_{i}\right|$ for $i>0$ (or $i \leq 0$ ) is equivalent to $\lambda_{2 i}$ (or $\lambda_{2 i+1}$ ) as shown by the coincided Spc-SVi and SVD-SVi curves in Fig. 1, so the spectral-derived $D s_{i}$ and $S D_{i}$ for $i>0$ (or $i \leq 0$ ) are equivalent to the SVD-computed $D s_{2 i}$ (or $D s_{2 i+1}$ ) and $S D_{2 i}$ (or $S D_{2 i+1}$ ), respectively. In view of the above explained and verified equivalences, we only need to present the results from the spectral formulations in the remaining part of this section.

Note that $\left|\gamma_{i} / \gamma_{0}\right|^{2} \leq 1.5 \times 10^{-5}, S g_{i} / S g \leq 1.29 \times 10^{-4}$, $D s_{i} / D s \leq 1.1 \times 10^{-7}$ and $S D_{i} / S D \leq 7.6 \times 10^{-5}$ when $|i| \geq$ 6 in Fig. 2 (or, equivalently, $i \geq 12$ in Fig. 1). Therefore, the upper limit of the wavenumber index $i$ can be truncated from $\mu_{+}=10$ to 5 for the summations (over $i>0$ ) in the spectral formulations of $S g, D s$ and $S D$ [see (2)-(4)] with virtually no loss of information. This implies that the 40 observations can be compressed into 10 uniformly spaced super-observations with the observation resolution coarsened from $\Delta x_{o}=3 \mathrm{~km}$ to $\Delta x_{s}=$ $4 \Delta x_{o}=12 \mathrm{~km}$ with no loss of information. This implication will be verified in Section 2.4.

\subsection{Information loss caused by uniform thinning}

According to the analysis in section 4.2 of X11, uniformly thinning the observations will always cause a loss of information and the information loss increases as the thinned observation resolution becomes increasingly coarse. The information losses for the signal and dispersion parts of the relative entropy and the Shannon entropy difference can be measured relative to $\operatorname{Sg}\left(\Delta x_{o}\right)$, $\operatorname{Ds}\left(\Delta x_{o}\right)$ and $S D\left(\Delta x_{o}\right)$, respectively, by

$\mathrm{SIL}=1-\operatorname{Sg}\left(\Delta x_{s}\right) / \operatorname{Sg}\left(\Delta x_{0}\right)$,

$\mathrm{DIL}=1-D s\left(\Delta x_{s}\right) / D s\left(\Delta x_{0}\right)$

and

$\mathrm{SDIL}=1=S D\left(\Delta x_{s}\right) / S D\left(\Delta x_{0}\right)$.

Here, $\Delta x_{s}\left(>\Delta x_{o}\right)$ denotes the thinned observation resolution; $S g\left(\Delta x_{o}\right), D s\left(\Delta x_{o}\right)$ and $S D\left(\Delta x_{o}\right)$ denote the information contents from the original observations; $S g\left(\Delta x_{s}\right), D s\left(\Delta x_{s}\right)$ and $S D\left(\Delta x_{s}\right)$ denote the information contents from the thinned observations. The relative information losses defined by SIL, DIL and SDIL in (12)-(14) may also be called information losses for short.

For the data described in Section 2.1, $\operatorname{Sg}\left(\Delta x_{o}\right), D s\left(\Delta x_{o}\right)$ and $S D\left(\Delta x_{o}\right)$ are computed from the spectral formulations in (2)-(4) with $\mu_{-}=-9$ and $\mu_{+}=10$ for $\mu \equiv \min (N, M)=20$. By reducing the number of observations from $M=40$ to $M_{s}=20$, 10,8 and 4 and thus reducing $\beta\left(\equiv M / N=\Delta x / \Delta x_{o}\right)=2$ to $\beta_{s}$ $\left(\equiv M_{s} / N=\Delta x / \Delta x_{s}\right)=1,1 / 2,2 / 5$ and $1 / 5$ in (2)-(4), $\operatorname{Sg}\left(\Delta x_{s}\right)$, $D s\left(\Delta x_{s}\right)$ and $S D\left(\Delta x_{s}\right)$ are computed for four types of thinned observations with the observation resolution $\Delta x_{o}$ coarsened to $\Delta x_{s}=2 \Delta x_{o}, 4 \Delta x_{o}, 5 \Delta x_{o}$ and $10 \Delta x_{o}$, respectively. The resulting SIL, DIL and SDIL calculated from (12)-(14) for the thinned observations (with $\Delta x_{s} / \Delta x_{o}=2,4,5$ and 10) are plotted in Fig. 3 by the thin solid curve labelled 'SIL-thin', light dotted curve labelled 'DIL-thin' and thin dashed curve labelled 'SDIL-thin', respectively. As shown, the information losses are significant and increase quasi-linearly with $\Delta x_{s} / \Delta x_{o}$. This exemplifies the analysis in section 4.2 of X11.

\subsection{Super-Obbing by spectral truncation}

According to the analysis in section 4.1 of X11, the original observations can be compressed into fewer super-observations by a proper spectral truncation with zero or minimum loss of information. This compression, called super-Obbing by spectral truncation, requires the observations to be uniformly distributed. The 


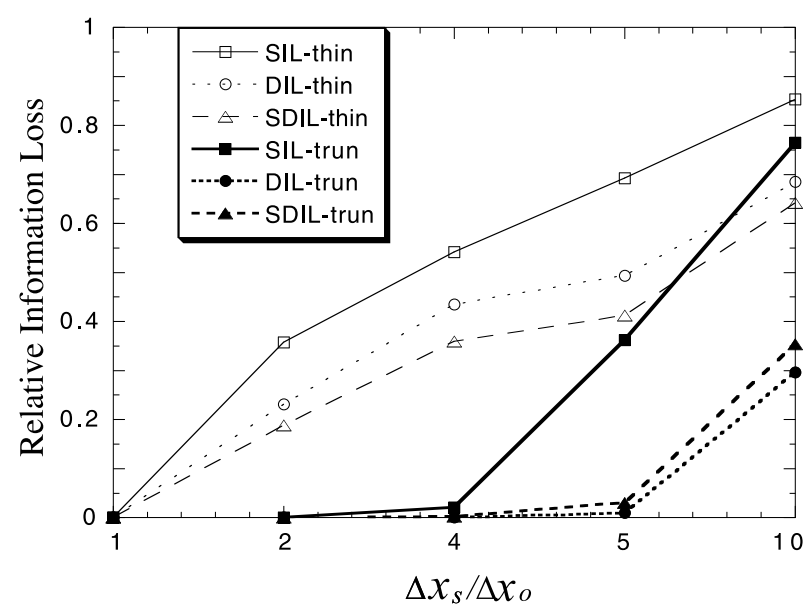

Fig. 3. Relative information losses SIL, DIL and SDIL caused by thinning plotted as functions of $\Delta x_{s} / \Delta x_{o}$ by the thin solid curve (labelled SIL-thin), light dotted curve (labelled DIL-thin") and thin dashed curve (labelled SDIL-thin), respectively; and relative information losses SIL, DIL and SDIL caused by spectral truncation plotted as functions of $\Delta x_{s} / \Delta x_{o}$ by the thick solid curve (labelled SIL-trun), dark dotted curve (labelled DIL-trun) and thick dashed curve (labelled SDIL-trun), respectively.

information losses caused by this super-Obbing can be measured by the same SIL, DIL and SDIL as those defined in (12)-(14) except that $\Delta x_{s}\left(>\Delta x_{o}\right)$ represents now the super-observation resolution, and thus $\operatorname{Sg}\left(\Delta x_{s}\right), D s\left(\Delta x_{s}\right)$ and $S D\left(\Delta x_{s}\right)$ denote the information contents from the super-observations. In this case, $\operatorname{Sg}\left(\Delta x_{o}\right), D s\left(\Delta x_{o}\right)$ and $S D\left(\Delta x_{o}\right)$ remain the same as those in Section 2.3, but $S g\left(\Delta x_{s}\right), D s\left(\Delta x_{s}\right)$ and $S D\left(\Delta x_{s}\right)$ are obtained for four types of super-observations with $\Delta x_{o}$ coarsened to $\Delta x_{s}=$ $2 \Delta x_{o}, 4 \Delta x_{o}, 5 \Delta x_{o}$ and $10 \Delta x_{o}$ by truncating the observation spectral space dimension from $M=40$ to $M_{s}=20,10,8$ and 4, respectively, (without changing $\beta$ ) as explained in section 4.1 of $\mathrm{X} 11$.

The information losses SIL, DIL and SDIL caused by the above super-observations are computed and plotted as functions of $\Delta x_{s} / \Delta x_{o}(=2,4,5$ and 10) in Fig. 3 by the thick solid curve labelled 'SIL-trun', dark dotted curve labelled 'DIL-trun' and thick dashed curve labelled 'SDIL-trun', respectively. As shown, the information losses are virtually zero for $\Delta x_{s} / \Delta x_{o}=$ 2 (because $\Delta x_{s}=\Delta x$ in this case) and remain negligibly small (=0.02, 0.0001 and 0.002 for SIL, DIL and SDIL, respectively) as $\Delta x_{s} / \Delta x_{o}$ increases to 4 . This verifies the implication stated at the end of Section 2.2. As $\Delta x_{s} / \Delta x_{o}$ further increases to 5 , the dispersion-part information loss DIL (or SDIL) remains small and below 0.01 (or 0.03), but the signal-part information loss SIL becomes significant and increases to 0.36 . Note that the signal-part information content depends on the sampled individual realization of the innovation and so does the signal-part information loss. Note also from in Fig. 1 that the Spc-Sgi curve reaches its peak value at the 4 th paired wavenumbers $k_{4}$ and $k_{-4}$ (corresponding to the singular-value indices at $i=8$ and 9 , respectively, in Fig. 1). When the wavenumber index increases from $i=0$ to $4,\left|\gamma_{i}\right|$ decreases rapidly from 11.84 to 1.01 but $\left|\gamma_{i}\right|^{4} /\left(1+\left|\gamma_{i}\right|^{2}\right)^{2}$ decreases slowly from 0.96 to 0.26 . Since $S g_{i}=$ $\left[\left|c_{i}\right|^{2} /\left(2 \beta S_{i}\right)\right]\left|\gamma_{i}\right|^{4} /\left(1+\left|\gamma_{i}\right|^{2}\right)^{2}$ for $N \leq M$ as shown in (6), the peak of the Spc-Sgi curve for $S g_{i} / S g$ can be largely explained by the peak of $\left|c_{i}\right|^{2} /\left(\beta S_{i}\right)$ at $|i|=4$, while the latter peak is tied up with the smallness of $S_{i}$ and local (secondary) peak of $\left|c_{i}\right|$ at $|i|=4$ in the innovation spectrum (not shown). When $\Delta x_{s} / \Delta x_{o}$ increases to 5 , the wavenumber $k_{4}$ is marginally retained but the wavenumber $k_{-4}$ is truncated. This truncation is significant and it explains why SIL increases rapidly (from 0.02 to 0.36 ) as $\Delta x_{s} / \Delta x_{o}$ increases from 4 to 5 in Fig. 3 .

Based on the equivalence between the spectral and generalized singular-value formulations explained and verified in Section 2.2, the above super-Obbing by spectral truncation can be viewed as a special and yet generalized form of super-Obbing by singular-value truncation (see section 4 of X07) for uniformly distributed observations. An advantage of this super-Obbing is that super-observations generated in the truncated spectral space can be easily transformed back and explicitly related to the original observations in the physical space. In particular, as shown in (41)-(42) of X11, super-observations can be expressed in the physical space by the original observations convoluted with a super-Obbing operator [see (40) of X11], and their error covariance can be given explicitly by the original observation error covariance convoluted with the super-Obbing operator. In this case, the original observations are required to be precisely uniformly distributed (in each direction) so that the spectral truncation can be accurately implemented for super-Obbing to avoid or minimize information loss. The required uniform distribution, however, can rarely be satisfied in real data assimilation, and this is a serious limitation for super-Obbing by spectral truncation, as commented at the end of section 4.1 of X11 and mentioned in the introduction of this paper.

\subsection{Super-Obbing by local averaging}

According to the analyses in sections 4.3 and 4.4 of X11, uniformly distributed observations with unbiased and spatially uncorrelated errors can be compressed into super-observations by local averaging with no loss of information until the superobservation resolution becomes coarser than the effective background resolution (Frehlich, 2008) or the background error covariance resolution (determined by $\pi / k_{c}$ where $k_{c}$ is the cutoff wavenumber of the background error power spectrum). In this case, the original observations are simply averaged over each $\Delta x_{s}$, where $\Delta x_{s}$ is the super-observation resolution. To verify and illustrate the above property, four types of superobservations are generated by local averaging with $\Delta x_{s}$ set to $\Delta x_{s}=2 \Delta x_{o}, 4 \Delta x_{o}, 5 \Delta x_{o}$ and $10 \Delta x_{o}$, respectively. The information losses caused by super-Obbing can be measured by the same SIL, DIL and SDIL as defined in (12)-(14) except that $S g\left(\Delta x_{s}\right)$, 


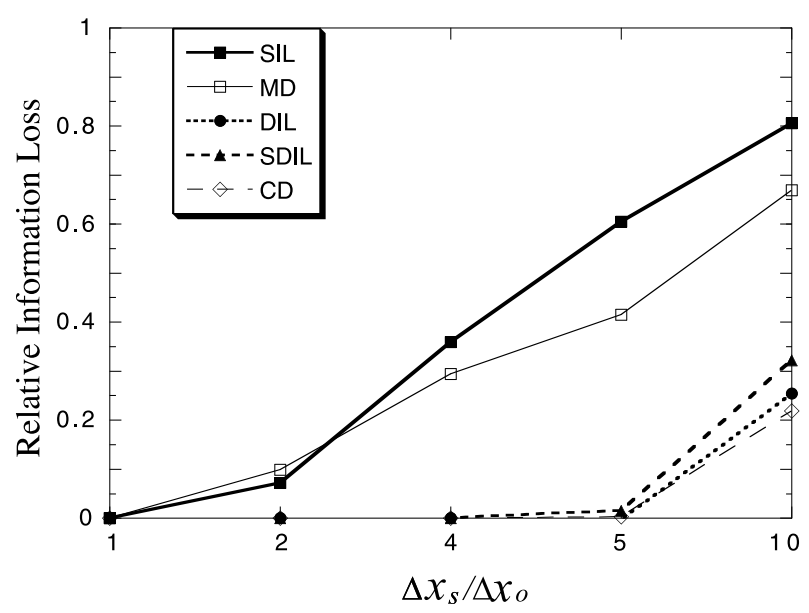

Fig. 4. Relative information losses SIL, DIL and SDIL caused by local averaging plotted as functions of $\Delta x_{s} / \Delta x_{o}$ by the dark solid, dark dashed and dark dotted curves, respectively; and relative degradations of the analysis mean and covariance, defined by $\mathrm{MD} / \mathrm{MD}_{0}$ and $\mathrm{CD} / \mathrm{CD}_{0}$, respectively, plotted as functions of $\Delta x_{s} / \Delta x_{o}$ by the thin solid curve (labelled MD) and thin dashed curve (labelled CD), respectively.

$D s\left(\Delta x_{s}\right)$ and $S D\left(\Delta x_{s}\right)$ are now the information contents from super-observations generated by local averaging. The superobservation error variance is estimated by $\sigma_{\mathrm{s}}^{2}=\sigma_{o}^{2} \Delta x_{o} / \Delta x_{s}$, where the sampling error in the original observations is assumed to remain constant and not yet start to increase with $\Delta x_{s}$ [see (43) and remark (ii) in section 4.4 of X11].

The computed SIL, DIL and SDIL are plotted in Fig. 4 by the thick solid curve, dark dotted curve and thick dashed curve, respectively. As shown, DIL and SDIL are zero for $\Delta x_{s} / \Delta x_{o}=$ 2 and remain negligibly small as $\Delta x_{s} / \Delta x_{o}$ increases to 5 , but SIL is non-zero (0.07) for $\Delta x_{s} / \Delta x_{o}=2$ and increases rapidly as $\Delta x_{s} / \Delta x_{o}$ increases beyond 2 . For the original observations, $D s\left(\Delta x_{o}\right)$ [or $\left.S D\left(\Delta x_{o}\right)\right]$ is the summation of $D s_{i}$ (or $S D_{i}$ ) over the wavenumber index range of $-9=\mu_{-} \leq i \leq \mu_{+}=10$, and $D s_{i}$ (or $S D_{i}$ ) is determined solely by $\left|\gamma_{i}\right|^{2}=\beta S_{i} / \sigma_{o}^{2}$. For $\Delta x_{s}=2 \Delta x_{o}$ $(=\Delta x)$, the number of super-observations is $M_{s}=20(=N)$, so $D s\left(\Delta x_{s}\right)$ [or $S D\left(\Delta x_{s}\right)$ is still the summation of $D s_{i}$ (or $S D_{i}$ ) over $-9 \leq i \leq 10$, and $D s_{i}$ (or $S D_{i}$ ) is still determined solely by $\left|\gamma_{i}\right|^{2}=$ $\beta_{s} S_{i} / \sigma_{s}^{2}=\beta S_{i} / \sigma_{0}^{2}$, where $\beta_{s} \equiv \Delta x / \Delta x_{s}$ and $\sigma_{s}^{2}=\sigma_{0}^{2} \Delta x_{o} / \Delta x_{s}$ are used. Thus, as revealed by the analysis in section 4.3 of $\mathrm{X} 11, D s\left(\Delta x_{s}\right)$ and $\left.S D\left(\Delta x_{s}\right)\right]$ remain the same as $D s\left(\Delta x_{o}\right)$ and $S D\left(\Delta x_{o}\right)$, respectively, for $\Delta x_{s}=2 \Delta x_{o}=\Delta x$. This explains why DIL and SDIL are zero for $\Delta x_{s} / \Delta x_{o}=2$ in Fig. 4 .

When $\Delta x_{s} / \Delta x_{o}$ increases to 5 , the number of superobservations reduces to $M_{s}=8(<N)$, so $D s\left(\Delta x_{s}\right)\left[\right.$ or $\left.S D\left(\Delta x_{s}\right)\right]$ is the summation of $D s_{i}$ (or $\left.S D_{i}\right)$ over $-3=\mu_{s-} \leq i \leq \mu_{s+}=4$, where $\mu_{s-} \equiv \operatorname{Int}\left[\left(1-\mu_{s}\right) / 2\right], \mu_{s+} \equiv \operatorname{Int}\left[\mu_{s} / 2\right]$ and $\mu_{s} \equiv \min (N$, $M_{s}$ ). Note that $D s_{i}$ (or $S D_{i}$ ) is determined solely by $\left|\gamma_{i}\right|^{2}=\beta_{s}$ $\Pi_{i} / \sigma_{s}^{2}=\beta \Pi_{i} / \sigma_{0}^{2}$, where $\Pi_{i}$ is the collapsed background error power spectrum. According to (25) of X11, we have $\Pi_{-3}=$
$S_{-3}+S_{5}, \Pi_{-2}=S_{-2}+S_{6}, \Pi_{-1}=S_{-9}+S_{-1}+S_{7}, \Pi_{0}=S_{-8}+$ $S_{0}+S_{8}, \Pi_{1}=S_{-7}+S_{1}+S_{9}, \Pi_{2}=S_{-6}+S_{2}+S_{10}, \Pi_{3}=$ $S_{-5}+S_{3}$, and $\Pi_{4}=S_{-4}+S_{4}$ for $N=20$ and $M_{s}=8$ (with $\left.\Delta x_{s}=5 \Delta x_{o}\right)$. Note also that $\left|\gamma_{i} / \gamma_{0}\right|^{2}=S_{i} / S_{o}$ for $M \geq N$, and $S_{i} / S_{o}$ decreases rapidly to $7.2 \times 10^{-} 3,4.5 \times 10^{-} 4,1.5 \times 10^{-} 5$ and virtually zero as $|i|$ increases from 0 to $4,5,6$ and beyond (as shown by the SVi2 curve in Fig. 2). Therefore, $S_{i} / S_{o}<$ or $\ll 4.5$ $\times 10^{-} 4$ for $|i|>4$, and we only need to consider the case of $|i|=$ 4. Note that $\Pi_{4}=S_{-4}+S_{4}=2 S_{4}$ due to the symmetry of $S_{-i}=$ $S_{i}$, so $S_{-4}$ is collapsed into $\Pi_{4}$ and this doubles $D s_{4}$ and $S D_{4}$. The original contribution of $D s_{-4}$ (or $\left.S D_{-4}\right)$ to $D s\left(\Delta x_{o}\right)$ [or $S D\left(\Delta x_{o}\right)$ ] in the summation over $-9 \leq i \leq 10$ for the original observations is thus largely retained by the doubled $D s_{4}\left(\right.$ or $\left.S D_{4}\right)$ in the summation over $-3 \leq i \leq 4$ for super-observations generated with $\Delta x_{s}=5 \Delta x_{o}$. This explains why the dispersion-part information loss, measured by DIL (or SDIL), remains negligibly small as $\Delta x_{s} / \Delta x_{o}$ increases to 5 in Fig. 4.

The signal-part information loss, measured by SIL, depends mainly on the difference between the original innovation spectrum $c_{i}$ and associated super-innovation spectrum, denoted by $c_{s i}$. The local averaging acts like a sinc-function filter in the spectral space [see the discussion of (40) in X11], so $\left|c_{s i}\right|$ is not exactly the same as $\left|c_{i}\right|$ even when $\Delta x_{s}=2 \Delta x_{o}=\Delta x$ [and the summation of $S g_{i}$ in (2) for the super-observations is still over the same range of $-9 \leq i \leq 10$ as for the original observations]. This explains why SIL is non-zero (0.07) for $\Delta x_{s} / \Delta x_{o}=$ 2 as shown in Fig. 4. When $\Delta x_{s} / \Delta x_{o}$ increases to 4, 5 and 10 , the number of super-observations reduces to $M_{s}=10,8$ and 4 , respectively. In this case, the difference between $\left|c_{s i}\right|$ and $\left|c_{i}\right|$ becomes increasingly large and the summation of $S g_{i}$ in (2) for the super-observations is confined to the successively narrowed ranges of $-4 \leq i \leq 5,-3 \leq i \leq 4$ and $-1 \leq i \leq 2$. This explains why SIL increases rapidly as $\Delta x_{s} / \Delta x_{o}$ increases to 4 and beyond as shown in Fig. 4.

As explained in section 5.1 of X07, the signal-part information loss measures the information loss caused by super-Obbing that degrades the analysis mean, and the analysis mean degradation (MD) can be quantified by

$\mathrm{MD}=\left|\mathbf{a}-\mathbf{a}_{s}\right|$,

where $\mathbf{a}$ and $\mathbf{a}_{s}$ are the state vectors of the analysis means obtained from the original observations and super-observations, respectively. On the other hand, the dispersion-part information loss measures the information loss caused by super-Obbing that degrades the analysis covariance and, the analysis covariance degradation (CD) can be quantified by

$\mathrm{CD}=\left\|\mathbf{A}-\mathbf{A}_{s}\right\|_{\mathrm{F}}$,

where $\mathbf{A}$ and $\mathbf{A}_{s}$ are the analysis covariance matrices obtained from the original observations and super-observations, respectively, and $\left\|\mathbf{A}-\mathbf{A}_{s}\right\|_{\mathrm{F}}$ denotes the Frobenius norm of $\mathbf{A}_{s}-\mathbf{A}$ defined by the square root of the sum of the squared absolute values of all the elements in $\mathbf{A}_{s}-\mathbf{A}$ [see (2.2-4) of Golub and Van 
Loan 1983]. If the observations are not used at all, then the analysis mean and covariance matrix reduce to the background mean and covariance matrix, denoted by $\mathbf{b}$ and $\mathbf{B}$, respectively. In this case, the degradations of the analysis mean and covariance reach their maxima $\mathrm{MD}_{0} \equiv|\mathbf{a}-\mathbf{b}|$ and $\mathrm{CD}_{0} \equiv\|\mathbf{A}-\mathbf{B}\|_{\mathrm{F}}$, respectively. The relative degradations of the analysis mean and covariance can be then measured by $\mathrm{MD} / \mathrm{MD}_{0}$ and $\mathrm{CD} / \mathrm{CD}_{0}$, respectively, and they are expected to increase with $\Delta x_{s} / \Delta x_{o}$ similarly to the relative information losses SIL and DIL (or SDIL), respectively. As shown in Fig. 4, these expected similarities are indeed verified by the closeness of the thin solid curve for $\mathrm{MD} / \mathrm{MD}_{0}$ to the thick solid curve for SIL and the closeness of the thin dashed curve for $\mathrm{CD} / \mathrm{CD}_{0}$ to the thick dotted curve for DIL (or thick dashed curve for SDIL).

\section{Applications to two-dimensional radar data on the conical surface of radar scan}

\subsection{Descriptions of the two-dimensional radar data and background field}

The two-dimensional radar data used in this section are the radial velocities on the conical surface at $0.75^{\circ}$ elevation angle scanned by the same phased array radar around 2108 UTC as described in Section 2.1. As these original radar data are dense and have high resolutions $(240 \mathrm{~m}$ in the radial direction and $1.6^{\circ}$ in the azimuthal direction) as mentioned in Section 2.1, the number of observations is quite large $(=10134)$ over the $\left(108 \times 60 \mathrm{~km}^{2}\right)$ area covered by the analysis domain on the conical surface at $0.75^{\circ}$ elevation angle. The original radar data are thinned to the coarsened resolutions of $\Delta r_{o}=1.2 \mathrm{~km}$ in the radial direction and $\Delta \theta_{o}=3.2^{\circ}$ in the azimuthal direction. After the thinning, the observation errors become essentially uncorrelated (Xu et al., 2007) to facilitate the tests in Section 3.3 for super-Obbing by local averaging to cause near-zero minimal information loss. The observation error variance is $\sigma_{0}^{2}=6.4 \mathrm{~m}^{2}$ $\mathrm{s}^{-2}$, as stated in Section 2.1. The analysis domain is a $108 \times 60$ $\mathrm{km}^{2}$ rectangular area on the conical surface at $0.75^{\circ}$ elevation angle, and it ranges from 30 to $138 \mathrm{~km}$ in $x$-direction and from -30 to $30 \mathrm{~km}$ in $y$-direction relative to the radar. The analysis grid consists of $18 \times 10$ grid boxes with a uniform resolution of $\Delta x=\Delta y=6 \mathrm{~km}$. The thinned radial-velocity data within the analyses domain are plotted in Fig. 5a. These thinned data will be used as the input observations for the analysis in this section, and the number of the observations is $M_{o}=1012$.

The background velocity field is produced by the COAMPS on the same $6 \mathrm{~km}$ grid as described in Section 2.1, and then projected onto the above two-dimensional analysis grid on the conical surface at $0.75^{\circ}$ elevation angle. The projected background radial-velocity field is shown in Fig. 5b. According to (5.1) of Xu and Gong (2003), the background radial-velocity error covariance between two points $\mathbf{x}_{2}$ and $\mathbf{x}_{1}$ on this surface can be modelled by

$B(\mathbf{r})=\cos (\Delta \beta) \sigma^{2} \exp \left[-r^{2} /\left(2 L^{2}\right)\right]$,

where $\mathbf{r}=\mathbf{x}_{2}-\mathbf{x}_{1}, r=|\mathbf{r}|, \Delta \beta$ is the angle between the two distance vectors represented by $\mathbf{x}_{2}$ and $\mathbf{x}_{1}$ in the radar-centred Cartesian coordinate system (see fig. 1 of $\mathrm{Xu}$ and Gong, 2003). Here, as in (1), $\sigma^{2}$ and $L$ denote the background radial-velocity error variance and decorrelation length scale, respectively. Again, as in Section 2.1, $\sigma^{2}=70 \mathrm{~m}^{2} \mathrm{~s}^{-2}$ and $L=15 \mathrm{~km}$ are selected for the illustrative purpose in this section.

\subsection{Applications of spectral formulations and singular-value formulations}

As shown in Fig. 5a, the input observations are not uniformly distributed and contain data-void areas. In this case, the signal part of the relative entropy cannot be easily or directly estimated by the two-dimensional spectral formulation in (29) of X11, because the signal part depends on the sampled observations and the observations must be properly mapped on a uniform grid in each direction in order to use the two-dimensional spectral formulation (which is an unexplored approach beyond this paper). However, the dispersion part of the relative entropy, $D s$, and the Shannon entropy difference, $S D$, do not depend on the individual realization of the sampled set of observations although they depend on the observation distribution and error covariance. Thus, $D s$ and $S D$ can be estimated approximately by the twodimensional spectral formulations in (30)-(31) of X11, as long as the input observations in Fig. 5a can be treated approximately as uniformly distributed with an averaged resolution in each direction and the background error covariance function in (17a) can be simplified into the following homogeneous and isotropic form:

$B(r)=\sigma^{2} \exp \left[-r^{2} /\left(2 L^{2}\right)\right]$.

The analysis domain in Fig. $5 \mathrm{~b}$ can be extended periodically as described in section 2.2 of X11. The simplified background error covariance function in (17b) can be extended periodically in each direction, similar to that in (1b), into the following form:

$$
\begin{aligned}
B(x, y)= & \left(\sigma^{2} / A_{0}\right) \sum_{i=-\infty}^{\infty} \sum_{j=-\infty}^{\infty} \\
& \times \exp \left\{-\left[\left(x-i D_{x}\right)^{2}+\left(y-j D_{y}\right)^{2}\right] /\left(2 L^{2}\right)\right\}
\end{aligned}
$$

where $A_{0}=\sum_{i=-\infty}^{\infty} \sum_{j=-\infty}^{\infty} \exp \left[-\left(i^{2} D_{x}^{2}+j^{2} D_{y}^{2}\right) /\left(2 L^{2}\right)\right]$ to ensure $B(0,0)=\sigma^{2}$. For the analysis grid described in Section 3.1, we have $\Delta x=\Delta y=6 \mathrm{~km}, N_{x}=18, N_{y}=10, D_{x}=$ $108 \mathrm{~km}, D_{y}=60 \mathrm{~km}$ and $A_{0}=1+6.7 \times 10^{-4}$ (with $L=15$ $\mathrm{km})$. The background error power spectrum can be derived from (17c) by using the generalized Fourier transformation in each direction similar to that in (10)-(11). The derived background 

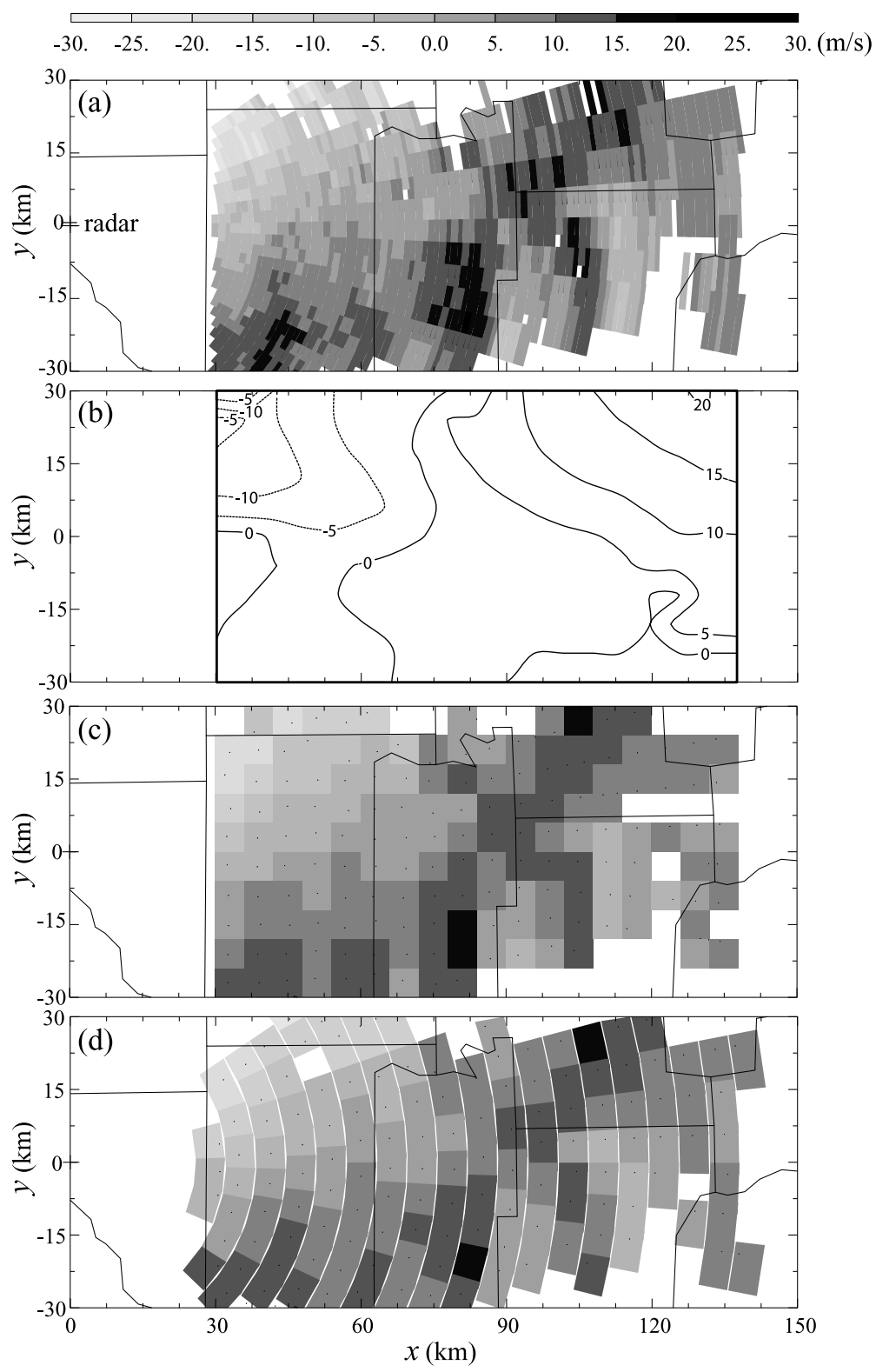

Fig. 5. (a) Input radial-velocity observations plotted by grey image pixels (with $\Delta r_{o}=1.2$ $\mathrm{km}$ and $\Delta \theta_{o}=3.2^{\circ}$ ) on the conical surface at $0.75^{\circ}$ elevation angle scanned by the radar, (b) background radial-velocity field plotted by contours of every $5 \mathrm{~m} \mathrm{~s}^{-1}$, (c) S1 super-observations plotted by grey image pixels (with $\Delta x_{s}=\Delta y_{s}=6 \mathrm{~km}$ ), and (d) S2 super-observations plotted by grey image pixels [with $\Delta r_{s}=6 \mathrm{~km}$ and $\Delta \theta_{s}=$ $\left.\left(\Delta r_{s} / r\right)\left(180^{\circ} / \pi\right)\right]$. The greyscale of every 5 $\mathrm{m} \mathrm{s}^{-1}$ is shown on the top. The $(x, y)-$ coordinate system is centred at the radar site. The + sign marks the radar site in panel (a) and the thin lines show the county boundaries in panels (a), (c) and (d). The dark rectangular boundary lines highlight the analysis domain in panel (b). The tiny black dot within each super-observation grey image pixel marks the location of the super-observation. error power spectrum has the following discrete form:

$$
\begin{aligned}
S_{i j}= & \left(\sigma^{2} / A_{0}\right) 2 \pi L^{2}(\Delta x \Delta y)^{-1} \exp \left[-\left(k_{i}^{2}+k_{j}^{2}\right) L^{2} / 2\right] \\
& \text { for } N_{x-} \leq i \leq N_{x+} \text { and } N_{y-} \leq j \leq N_{y+},
\end{aligned}
$$

where $k_{i}=i \Delta k_{x} k_{j}=j \Delta k_{y}, \Delta k_{x} \equiv 2 \pi / D_{x}, \Delta k_{y} \equiv 2 \pi / D_{y}, D_{x}=$ $N_{x} \Delta x, D_{y}=N_{y} \Delta y, N_{x-} \equiv \operatorname{Int}\left[\left(1-N_{x}\right) / 2\right], N_{x+} \equiv \operatorname{Int}\left[N_{x} / 2\right]$, $N_{y-} \equiv \operatorname{Int}\left[\left(1-N_{y}\right) / 2\right]$ and $N_{y+} \equiv \operatorname{Int}\left[N_{y} / 2\right]$.

For the input observations in Fig. 5a, the averaged resolutions can be represented by $\Delta x_{o}=1.2 \mathrm{~km}$ in the $x$ direction and $\Delta y_{o}=$ $5 \mathrm{~km}$ in the $y$ direction. With these averaged resolutions, the observation space can be approximated by a $M_{x} \times M_{y}=90 \times 12$ uniform grid over the $108 \times 60 \mathrm{~km}^{2}$ rectangular area of the above analysis domain. The observation error covariance is $\sigma_{0}^{2}$ multiplied by an identity matrix in the approximated observation space of $R^{M}=R^{M x} \otimes R^{M y}$ where $M=M_{x} \times M_{y}=90 \times 12$. The observation error power spectrum is thus a constant in the approximated observation-spectral space and is given by

$C_{i j}=C\left(i \Delta k_{x}, j \Delta k_{y}\right)=\sigma_{0}^{2}$

$$
\text { for } M_{x-} \leq i \leq M_{x+} \text { and } M_{y-} \leq j \leq M_{y+} \text {, }
$$

where $M_{x-} \equiv \operatorname{Int}\left[\left(1-M_{x}\right) / 2\right], M_{x+} \equiv \operatorname{Int}\left[M_{x} / 2\right], M_{y-} \equiv \operatorname{Int}[(1-$ $\left.\left.M_{y}\right) / 2\right]$ and $M_{y+} \equiv \operatorname{Int}\left[M_{y} / 2\right]$.

By substituting the derived background error power spectrum in (18) and approximated observation error power spectrum in 
Table 1. Spectral-estimated and SVD-computed information contents from the input observations in Fig. 5a.

\begin{tabular}{lccc}
\hline & $S g$ & $D s$ & $S D$ \\
\hline Spectral-estimated & N/A & 57.0 & 75.2 \\
SVD-computed with B(r) in (17a): & 66.7 & 61.6 & 83.6 \\
SVD-computed with B $(r)$ in $(17 \mathrm{~b}):$ & 70.2 & 58.4 & 78.7 \\
SVD-computed with B $(x, y)$ in $(17 \mathrm{c}):$ & 83.9 & 53.5 & 71.0 \\
\hline
\end{tabular}

(19) into the spectral formulations in (30)-(32) of X11, we can estimate $D s$ and $S D$ approximately for the dispersion part of the information content extracted from the input observations in Fig. 5a by the analysis with the background field in Fig. 5b. As listed in the first row of Table 1, the estimated values are $D s=57.0$ and $S D=75.2$, while the observation space dimension is approximated by $M=M_{x} \times M_{y}=90 \times 12=$ 1080. These spectral-estimated values are close to their counterpart values computed by the singular-value formulations, called SVD-computed values, as shown below.

With the singular-value formulations [see (3.6)-(3.10) of $\mathrm{X} 07]$, the background error covariance matrix $\mathbf{B}$ can be constructed directly by discretizing $B(\mathbf{r})$ in $(17 \mathrm{a}), B(r)$ in $(17 \mathrm{~b})$ or $B(x, y)$ in $(17 \mathrm{c})$ on the analysis grid, while the observation error covariance matrix $\mathbf{R}$ is simply given by $\sigma_{0}^{2} \mathbf{I}_{M o}$, where $\mathbf{I}_{M o}$ is an identity matrix in the observation space $R^{M o}$ and $M_{o}$ (=1012) is the number of the input observations in Fig. 5a. The innovation vector, denoted by $\mathbf{d} \in R^{M o}$, in the observation space can be obtained by subtracting the background values in Fig. 5b from the observations in Fig. 5a. By substituting this $\mathbf{d}$ with the above constructed $\mathbf{B}$ and $\mathbf{R}$ into (3.6) of X07, we can compute $S g$ for the signal part of the information content from the input observations in Fig. 5a. As listed in the first column of Table 1, the computed value is $S g=66.7$ for $\mathbf{B}$ constructed from the original $B(\mathbf{r})$ in (17a), and the value is inflated slightly (by $5.2 \%$ ) to 70.2 when $\mathbf{B}$ is constructed from the simplified $B(r)$ in (17b), and inflated further (by 19.5\%) to 83.9 when $\mathbf{B}$ is constructed from the periodically extended $B(x, y)$ in $(17 \mathrm{c})$. By substituting the above $\mathbf{B}$ and $\mathbf{R}$ into (3.9)-(3.10) of X07, we can compute $D s$ and $S D$ for the input observations in Fig. 5a. As listed in Table 1 , the computed values are $D s=61.6$ and $S D=83.6$ when $\mathbf{B}$ is constructed from the original $B(\mathbf{r})$ in (17a), and the values are deflated slightly (by $5.2 \%$ and $5.9 \%$ ) to $D s=58.4$ and $S D=78.7$, respectively, when $\mathbf{B}$ is constructed from $B(r)$ in (17b), and deflated further (by $2.4 \%$ and $9.8 \%$ ) to $D s=53.5$ and $S D=71.0$, respectively, when $\mathbf{B}$ is constructed from $B(x, y)$ in $(17 \mathrm{c})$.

Note that $\cos (\Delta \beta)(\leq 1)$ is a non-isotropic damping factor for $B(\mathbf{r})$ in (17a). When this factor is neglected by the simplified $B(r)$ in (17b), the spatial correlation is enhanced in $B(r)$ relative to that in $B(\mathbf{r})$. When $B(r)$ is extended periodically into $B(x, y)$ in (17c), the spatial correlation is further enhanced. Thus, when $\mathbf{B}$ is constructed from $B(r)$ in place of $B(\mathbf{r})$ and then from $B(x, y)$ in place of $B(r)$, the off-diagonal elements of $\mathbf{B}$ are inflated consecutively but the diagonal elements still have the constant value of $\sigma^{2}$. In this case, the sum of $\lambda_{i}^{2}$ is not changed [because $\sum_{i=1}^{N} \lambda_{i}^{2}=$ $\operatorname{trace}\left(\mathbf{M M}^{\mathrm{T}}\right)=\operatorname{trace}\left(\mathbf{M}^{\mathrm{T}} \mathbf{M}\right)=\operatorname{trace}(\mathbf{B}) / \sigma_{0}^{2}=N \sigma^{2} / \sigma_{0}^{2}$ for $N=$ $N_{x} \times N_{y}=18 \times 10<M_{o}=1012$ and $\mathbf{R}=\sigma_{0}^{2} \mathbf{I}_{M o}$ where $\mathbf{H}^{\mathrm{H}} \mathbf{H}=$ $\mathbf{I}_{N}$ is used], but $\lambda_{i}^{2}$ (that is, the eigenvalue of $\mathbf{M}^{\mathrm{T}} \mathbf{M}=\mathbf{B} / \sigma_{0}^{2}$ ) is a consecutively steepened decreasing function of $i$ when $\mathbf{B}$ is constructed from $B(r)$ in place of $B(\mathbf{r})$ and then from $B(x, y)$ in place of $B(r)$. The incremental change of $\lambda_{i}^{2}$ caused by each consecutive steepening is thus a zero-sum function of $i$, and this function increases positively (or negatively) as $i$ decreases toward 1 (or increases toward $N$ ). This zero-sum incremental change of $\lambda_{i}^{2}$ explains why the SVD-computed $D s$ and $S D$ are deflated consecutively when $\mathbf{B}$ is constructed from $B(r)$ in place of $B(\mathbf{r})$ and then from $B(x, y)$ in place of $B(r)$, as shown in the last three rows of Table 1 .

The $i$ th component of $S g$ is computed by $S g_{i}=$ $d_{i}^{\prime 2} / \lambda_{i}^{2}\left(1+\lambda_{i}^{2}\right)^{-2} / 2$ where $d^{\prime}{ }_{i}$ is the $i$ th component of the transformed innovation vector $\mathbf{d}^{\prime}=\mathbf{U}^{\mathrm{T}} \mathbf{R}^{-1 / 2} \mathbf{d}$ in the singular-vector space [see (3.6) of X07]. For $\mathbf{d}$ computed from the observations in Fig. 5a and the background field in Fig. 5b, the transformed innovation vector $\mathbf{d}^{\prime}$ has its large-amplitude (measured by $\left|d^{\prime}{ }_{i}\right|$ ) components mostly within the index range of $1 \leq i<N / 2$, and this feature becomes increasingly distinctive when $\mathbf{B}$ is constructed from $B(r)$ in place of $B(\mathbf{r})$ and then from $B(x, y)$ in place of $B(r)$. This feature in combination with the aforementioned zero-sum incremental change of $\lambda_{i}^{2}$ explains why $S g$ is inflated consecutively when $\mathbf{B}$ is constructed from $B(r)$ in place of $B(\mathbf{r})$ and then from $B(x, y)$ in place of $B(r)$, as shown in the first column of Table 1 .

The spectral-estimated $D s$ (or $S D$ ) in the first row of Table 1 can be verified against the SVD-computed $D S$ (or $S D$ ) in the last row of Table 1, because they both use the periodically extended background error covariance $B(x, y)$ in $(17 \mathrm{c})$. When the observation error power spectrum is derived in (19), the observations are assumed to cover the analysis domain uniformly with no datavoid area. The observation space dimension is approximated by $M=M_{x} \times M_{y}=90 \times 12=1080$ in the derivation of (19) which is slightly larger than that $\left(M_{o}=1012\right)$ for the input observations in Fig. 5a, while the observation error variance remains the same $\left(\sigma_{0}^{2}=6.4 \mathrm{~m}^{2} \mathrm{~s}^{-2}\right)$. This slightly increased number of observations (with the same $\sigma_{0}^{2}$ ) explains why the spectral-estimated $D s$ (or $S D$ ) in the first row is slightly larger than the SVD-computed $D s$ (or $S D$ ) in the last row of Table 1. Consequently, the spectralestimated $D s$ (or $S D$ ) is very close to and only slightly smaller, by merely $4.4 \%$ (or $2.4 \%$ ), than the SVD-computed $D s$ (or $S D$ ) with $\mathbf{B}$ constructed from $B(r)$ in the third row of Table 1. Therefore, $B(r)$ will be used as an intermediate approximation of the original $B(\mathbf{r})$, which is between $B(x, y)$ and $B(\mathbf{r})$, to compute the information losses caused by super-observations and verify the spectral-estimated information losses in the next subsection. 
Table 2. Super-observation resolution $\Delta l_{s}$, its ratio $\Delta l_{s} / \Delta l_{o}$ to the input observation resolution $\left(\Delta l_{o}=1.2 \mathrm{~km}\right)$ and its associated super-observation space dimension $M_{S}$ listed (in the first five rows) for each set (in each column) of S1 super-observations, S2 super-observations and spectral-approximated super-observations. The last two rows list the spectral-approximated super-observation resolutions $\left(\Delta x_{s}, \Delta y_{s}\right)$.

\begin{tabular}{lcrlllc}
\hline$\Delta l_{s}(\mathrm{~km})$ & 3 & 6 & 12 & 15 & 24 & 30 \\
$\Delta l_{s} / \Delta l_{o}$ & 2.5 & 5 & 10 & 12.5 & 20 & 25 \\
$\mathrm{~S} 1 M_{s}$ & 410 & 153 & 44 & 30 & 15 & 8 \\
$\mathrm{~S} 2 M_{s}$ & 337 & 136 & 50 & 34 & 19 & 14 \\
Spectral $M_{s}$ & 432 & 180 & 45 & 32 & 15 & 8 \\
Spectral $\Delta x_{s}(\mathrm{~km})$ & 3 & 6 & 12 & 13.5 & 21.6 & 27 \\
Spectral $\Delta y_{s}(\mathrm{~km})$ & 5 & 6 & 12 & 15 & 24 & 30 \\
\hline
\end{tabular}

\subsection{Super-Obbing by local averaging}

In this subsection, two types of super-observations, called S1 and $S 2$, are generated from the input observations in Fig. 5a by local averaging. Each $\mathrm{S} 1$ super-observation covers a square area of $\Delta x_{s} \times \Delta y_{s}$ with $\Delta x_{s}=\Delta y_{s}$ set to a prescribed resolution, denoted by $\Delta l_{s}$. Each S2 super-observation covers a sector area with the radial-range interval $\Delta r_{s}$ set to a prescribed resolution $\Delta l_{s}$ in the radar-centred polar coordinate system (on the conical surface of radar scan), while the azimuth span of the sector area is determined by $\Delta \theta_{s}=\left(\Delta r_{s} / r\right)\left(180^{\circ} / \pi\right)$ where $r$ is the radial range distance from the radar to the far side of the sector area. Each super-observation of either type is computed by averaging the input observations locally in its covered area. The location of each super-observation is assigned to the centroid of the input data points in its covered local area. The error variance of each super-observation is estimated by $\sigma_{s}^{2}=\sigma_{0}^{2} / n_{s}$, where $n_{s}$ is the number of input observations in the super-observation covered local area and the input observation error variance $\sigma_{0}^{2}$ is assumed to be constant and not affected by the increase of $\Delta x_{s}$ [see (43) and remark (ii) in section 4.4 of X11].

Six sets of $\mathrm{S} 1$ super-observations are generated with $\Delta x_{s}=$ $\Delta y_{s}=\Delta l_{s}$ prescribed to $3,6,12,15,24$ and $30 \mathrm{~km}$, respectively, as listed in the first row of Table 2 . The associated ratios with respect to the input observation resolution $\Delta x_{o}=\Delta l_{o}(=1.2$ $\mathrm{km}$ ) in the $x$-direction are $\Delta l_{s} / \Delta l_{o}=2.5,5,10,12.5,20$ and 25, respectively, as listed in the second row of Table 2 . These six sets contain $M_{s}=410,153,44,30,15$ and 8 super-observations, respectively, as listed in the third row of Table 2, and an example is shown in Fig. $5 \mathrm{c}$ for the set obtained with $\Delta x_{s}=\Delta y_{s}=\Delta l_{s}=6$ $\mathrm{km}$. Six sets of S2 super-observations are generated with $\Delta r_{s}=$ $\Delta l_{s}$ prescribed to $3,6,12,15,24$ and $30 \mathrm{~km}$, respectively, as listed again in the first row of Table 2 . The associated ratios with respect to $\Delta l_{o}$ are the same as those listed in the second row of Table 2. These six sets contain $M_{s}=337,136,50,34,19$ and 14 super-observations, respectively, as listed in the fourth row of Table 2, and an example is shown in Fig. 5d for the set obtained with $\Delta r_{s}=\Delta l_{s}=6 \mathrm{~km}$. As shown in Fig. 5c (or 5d), super-observation areas do not overlap and their combined area covers all the input data points in Fig. 5a.

For each set of S1 or S2 super-observations listed in Table 2, $S g$, Ds and $S D$ can be computed by the singular-value formulations in the same way as described in the previous subsection for the input observations. The background error covariance matrix $\mathbf{B}$ is still constructed directly from either $B(\mathbf{r})$ in (17a) or $B(r)$ in (17b), while the super-observation error covariance matrix $\mathbf{R}$ is simply given by $\sigma_{s}^{2} \mathbf{I}_{M s}$ (since the super-observation errors are still spatially uncorrelated) where $\mathbf{I}_{M s}$ is an identity matrix in the super-observation space $R^{M s}$ and $M_{s}$ is the number of superobservations in the concerned set. The super-innovation vector, $\mathbf{d} \in R^{M s}$, is obtained by subtracting the background values in Fig. $5 b$ from the super-observations in the concerned set. By substituting the above d, $\mathbf{B}$ and $\mathbf{R}$ into (3.6) and (3.9)-(3.10) of X07, $S g, D s$ and $S D$ are obtained for each set of S1 or S2 super-observations with $\mathbf{B}$ constructed from either $B(\mathbf{r})$ or $B(r)$.

The relative information losses caused by each set of superobservations with respect to the input observations in Fig. 5a can be measured and computed by SIL, DIL and SDIL in the same way as those in (12)-(14) except that $S g\left(\Delta x_{o}\right), D s\left(\Delta x_{o}\right)$ and $S D\left(\Delta x_{o}\right)$ are replaced by $\operatorname{Sg}\left(\Delta l_{o}\right), D s\left(\Delta l_{o}\right)$ and $S D\left(\Delta l_{o}\right)$, respectively, to denote the information contents from the input observations in Fig. 5a, and $S g\left(\Delta x_{s}\right), D s\left(\Delta x_{s}\right)$ and $S D\left(\Delta x_{s}\right)$ are replaced by $S g\left(\Delta l_{s}\right), D s\left(\Delta l_{s}\right)$ and $S D\left(\Delta l_{s}\right)$, respectively, to denote the information contents from each set of super-observations listed in Table 2. Here, $\Delta l_{o}$ represents $\left(\Delta r_{o}, \Delta \theta_{o}\right)$ with $\Delta r_{o}=\Delta l_{o}$ $(=1.2 \mathrm{~km})$ and $\Delta \theta_{o}=3.2^{\circ}$ for the input observations in Fig. 5a, and $\Delta l_{s}$ represents $\left(\Delta x_{s}, \Delta y_{s}\right)$ with $\Delta x_{s}=\Delta y_{s}=\Delta l_{s}$ for $\mathrm{S} 1$ super-observations or represents $\left(\Delta r_{s}, \Delta \theta_{s}\right)$ with $\Delta r_{s}=\Delta l_{s}$ and $\Delta \theta_{s}=\left(\Delta l_{s} / r\right)\left(180^{\circ} / \pi\right)$ for S2 super-observations.

The SVD-computed SIL and DIL for the six sets of S1 (or S2) super-observations with $\mathbf{B}$ constructed from $B(\mathbf{r})$ in (17a) are plotted in Fig. 6 as functions of $\Delta l_{s} / \Delta l_{o}$ by the dark longdashed (or dark short-dashed) curve labelled SIL-S1a (or SILS2a) and dark dot-dashed (or dark dotted) curves labelled DILS1a (or DIL-S2a), respectively. The counterpart SIL and DIL computed with $\mathbf{B}$ constructed from $B(r)$ in $(17 \mathrm{~b})$ are plotted in Fig. 6 by the grey long-dashed (or grey short-dashed) curve labelled SIL-S1b (or SIL-S2b) and grey dot-dashed (or grey dotted) curve labelled DIL-S1b (or DIL-S2b), respectively. The SVD-computed SDIL curves (not shown) are very similar to and only slightly higher than their counterpart DIL curves in Fig. 6. Note that $\Delta l_{s} / \Delta l_{o}=1$ corresponds to the case of no superObbing and thus no information loss, so all the curves start from zero at $\Delta l_{s} / \Delta l_{o}=1$ in Fig. 6 . All the grey curves are very close to their counterpart dark curves, so the simplified $B(r)$ in (17b) can be indeed used as an approximation of the original $B(\mathbf{r})$ in (17a) to facilitate the use of the spectral formulation to estimate the information losses as shown below.

When the spectral formulations in (30)-(32) of X11 are used to estimate $D s$ (or $S D$ ) for each set of super-observations, the super-observation space is approximated by a $M_{x s} \times M_{y s}$ 


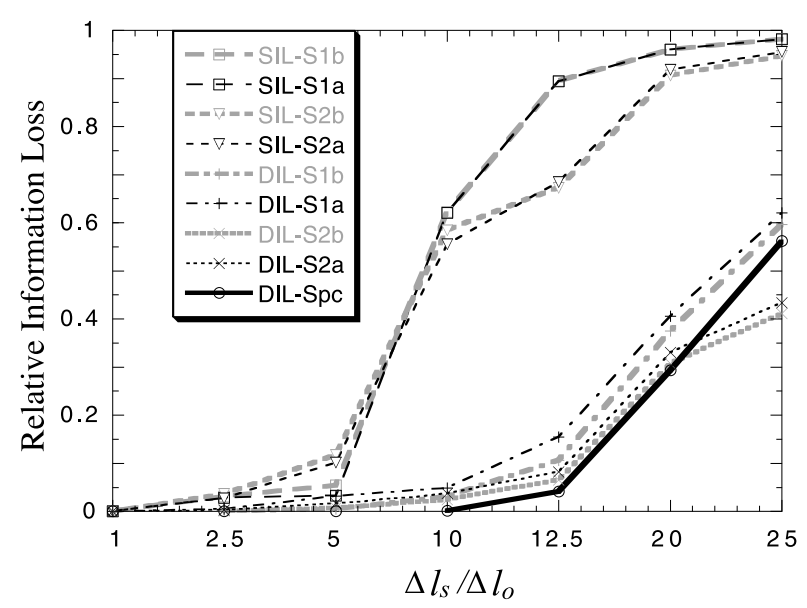

Fig. 6. SVD-computed SIL and DIL for S1 (or S2) super-observations with $\mathbf{B}$ constructed from $B(\mathbf{r})$ in (17a) plotted as functions of $\Delta l_{s} / \Delta l_{o}$ by the dark long-dashed (or dark short-dashed) curve labelled SIL-S1a (or SIL-S2a) and dark dot-dashed (or dark dotted) curves labelled DIL-S1a (or DIL-S2a), respectively; SVD-computed SIL and DIL for $\mathrm{S} 1$ (or S2) super-observations with $\mathbf{B}$ constructed from $B(r)$ in (17b) plotted as functions of $\Delta l_{s} / \Delta l_{o}$ by the grey long-dashed (or grey short-dashed) curve labelled SIL-S1b (or SIL-S2b) and grey dot-dashed (or grey dotted) curve labelled DIL-S1b (or DIL-S2b), respectively; and spectral-estimated DIL plotted as a function of $\Delta l_{s} / \Delta l_{o}$ by the dark solid curve labelled DIL-Spc. See the first two rows and last two row in Table 2 for the super-observation resolutions $\Delta l_{s}$ and $\left(\Delta x_{s}, \Delta y_{s}\right)$ associated with $\Delta l_{s} / \Delta l_{o}$.

uniform grid over the $D_{x} \times D_{y}=108 \times 60 \mathrm{~km}^{2}$ rectangular area of the analysis domain, where $M_{x s}=\operatorname{Int}\left[D_{x} / \Delta x_{s}+1 / 2\right]$ and $M_{y s}=\operatorname{Int}\left[D_{y} / \Delta y_{s}+1 / 2\right]$. For the six sets of S1 or S2 super-observations, the super-observation space dimensions are approximated by $M_{s}=M_{x s} \times M_{y s}=36 \times 12=432,18 \times 10=$ $180,9 \times 5=45,8 \times 4=32,5 \times 3=15$ and $4 \times 2=8$, respectively, as listed in the fifth row of Table 2 . The approximated super-observation resolutions are $\left(\Delta x_{s}, \Delta y_{s}\right)=(3,5)$, $(6,6),(12,12),(13.5,15),(21.6,24)$ and $(27,30) \mathrm{km}$, respectively, as listed in the last two rows of Table 2. In this case, the background error power spectrum is still given by (18), but the observation error power spectrum in (19) is replaced by the following super-observation error power spectrum

$$
\begin{aligned}
C_{i j}= & C\left(i \Delta k_{x}, j \Delta k_{\mathrm{y}}\right)=\sigma_{\mathrm{s}}^{2}=\sigma_{\mathrm{s}}^{2} / n_{\mathrm{s}} \\
& \text { for } M_{x s-} \leq i \leq M_{x s+} \text { and } M_{y s-} \leq j \leq M_{y s+},
\end{aligned}
$$

where $n_{s}=\Delta x_{s} \Delta y_{s} /\left(\Delta x_{o} \Delta y_{o}\right)$ is the averaged number of input observations in the local area covered by a super-observation, $M_{x s}=\operatorname{Int}\left[\left(1-M_{x s}\right) / 2\right], M_{x s+} \equiv \operatorname{Int}\left[M_{x s} / 2\right], M_{y s-} \equiv \operatorname{Int}[(1-$ $\left.\left.M_{y s}\right) / 2\right]$ and $M_{y s+} \equiv \operatorname{Int}\left[M_{y s} / 2\right]$. By substituting (18) and (20) into (30)-(32) of X11, we can obtain the spectral-estimated Ds (or $S D$ ) for each set of super-observations and then estimate the associated information loss, DIL (or SDIL), relative to the spectral-estimated $D s$ (or $S D$ ) for the input observations (listed in the first row of Table 1). The spectral-estimated DIL is plotted as functions of $\Delta l_{s} / \Delta l_{o}$ by the dark solid curve labelled DILSpc in Fig. 6, where $\Delta l_{o}$ represents $\left(\Delta x_{o}, \Delta y_{o}\right)$ with $\Delta x_{o}=$ $\Delta l_{o}=1.2 \mathrm{~km}$ and $\Delta y_{o}=5 \mathrm{~km}$ for the approximated observation resolutions used in the derivation of (19), and $\Delta l_{s}$ represents $\left(\Delta x_{s}, \Delta y_{s}\right)$, with $\Delta x_{s}=\Delta l_{s}$ for $\Delta l_{s} \leq 12 \mathrm{~km}$ and $\Delta y_{s}=\Delta l_{s}$ for $\Delta l_{s}>12 \mathrm{~km}$, as shown by the approximated super-observation resolutions listed in the last two rows of Table 2. As shown in Fig. 6, the spectral-estimated DIL-Spc curve follows closely the SVD-computed DIL-S1b curve with $\mathbf{B}$ constructed from $B(r)$.

All the curves in Fig. 6 increase with $\Delta l_{s} / \Delta l_{o}$ monotonically, and the SVD-computed SIL curves increase more rapidly than the SVD-computed DIL curves especially when $\Delta l_{s} / \Delta l_{o}$ increases from 5 to 10 and beyond. Note that $\Delta x_{o}=1.2 \mathrm{~km}$ and $\Delta l_{s} / \Delta l_{o}=5$ corresponds to $\Delta x_{s}=\Delta y_{s}=\Delta l_{s}=6 \mathrm{~km}$ (or $\Delta r_{s}=\Delta l_{s}=6 \mathrm{~km}$ ) for the $\mathrm{S} 1$ (or $\mathrm{S} 2$ ) super-observations, so the super-observation resolution becomes the same as the background resolution $(\Delta x=\Delta y=6 \mathrm{~km})$ when $\Delta l_{s} / \Delta l_{o}=5$ in Fig. 6. For the one-dimensional observations in Section 2, $\Delta x_{o}=$ $3 \mathrm{~km}$ and the super-observation resolution becomes the same as the background resolution $(\Delta x=6 \mathrm{~km})$ when $\Delta x_{s} / \Delta x_{o}=2$ in Fig. 4. Thus, the rapid increase of SIL after $\Delta l_{s} / \Delta l_{o}>5$ in Fig. 6 is similar to the rapid increase of SIL (shown by the dark solid curve) after $\Delta x_{S} / \Delta x_{o}>2$ in Fig. 4 . This rapid increase of SIL can be explained by the reduced super-observation space dimension (below the background space dimension) plus the reduced amplitude of the transformed super-innovation vector caused by the local averaging (that acts like a sinc-function filter in the spectral space), which is similar to that explained for the one-dimensional super-observations in Section 2.5.

As shown in Fig. 6, the SVD-computed DIL curves increase slowly from zero to various levels below 0.05 when $\Delta l_{s} / \Delta l_{o}$ increases from 1 to 10 . Note that $\Delta l_{s} / \Delta l_{o}=10$ corresponds to $\Delta x_{s}=\Delta y_{s}=12 \mathrm{~km}$ (or $\Delta r_{s}=12 \mathrm{~km}$ ) for the S1 (or S2) superobservations and this super-observation resolution $(=12 \mathrm{~km})$ is still finer than the background error de-correlation length scale $L=15 \mathrm{~km}$. This explains why the SVD-computed DIL curves all remain very low $(<0.05)$ for $\Delta l_{s} / \Delta l_{o} \leq 10$ in Fig. 6. Note also that the $\mathrm{S} 1$ and $\mathrm{S} 2$ super-observations are not exactly uniformly distributed (because the location of each super-observation is assigned to the centroid of the input data points in its covered local area as described in Section 3.1 and shown in Figs. 5c and d), and they both contain data-void areas due to the incomplete coverage of the input data as shown in Fig. 5. This explains why the SVD-computed DIL curves increase slowly but notably above zero as $\Delta l_{s} / \Delta l_{o}$ increases from 1 to 10 in Fig. 6. On the other hand, the spectral-estimated DIL curve (dark solid labelled DIL-Spc) remains virtually zero for $\Delta l_{s} / \Delta l_{o} \leq 10$ in Fig. 6 . Similarly, the dark dotted DIL curve computed for the uniform one-dimensional super-observations is also virtually zero for $\Delta x_{s} / \Delta x_{o} \leq 5$ as shown in Fig. 4 , and this is simply because $\Delta x_{s} / \Delta x_{o}=5$ in Fig. 4 and $\Delta l_{s} / \Delta l_{o}=10$ in Fig. 6 both correspond to the same super-observation resolution of $\Delta x_{s}=12 \mathrm{~km}$ and thus the same ratio of $\Delta x_{s} / L(=0.8)$. 
As listed in Table 2, the number of S1 super-observations is larger (or smaller) than that of S2 super-observations for $\Delta l_{s} / \Delta l_{o} \leq 5$ (or $\Delta l_{s} / \Delta l_{o} \geq 10$ ). This explains why the SILS1 curves are lower (or higher) than their respective SIL-S2 curves for $\Delta l_{s} / \Delta l_{o} \leq 5$ (or $\Delta l_{s} / \Delta l_{o} \geq 10$ ) as shown in Fig. 6 . Note also that the number of $S 1$ super-observations is substantially smaller than that of S2 super-observations for $\Delta l_{s} / \Delta l_{o} \geq$ 20) as listed in Table 2. This explains why the DIL-S1 curves become significantly higher than their respective DIL-S2 curves when $\Delta l_{s} / \Delta l_{o} \geq 20$ as shown in Fig. 6. Also as listed in Table 2, the number of super-observations approximated by $M_{s}=M_{x s} \times$ $M_{y s}$ for the super-observation error power spectrum in (20) is much closer to the number of S1 super-observations than the number of S2 super-observations and even becomes identical to the number of $\mathrm{S} 1$ super-observations when $\Delta l_{s} / \Delta l_{o} \geq 20$. This explains why the spectral-estimated DIL curve follows the SVDcomputed DIL-S1b curve more closely than the DIL-S2b curve especially when $\Delta l_{s} / \Delta l_{o} \geq 20$, as shown in Fig. 6. A similar close comparison is seen between the spectral-estimated SDIL curve and the SVD-computed SDIL-S1b curve (not shown) for the $\mathrm{S} 1$ observations with $\mathbf{B}$ constructed from $B(r)$ in (17b). These close comparisons indicate that the spectral formulations can be used to estimate the information loss caused by local averaging for the purpose of finding an optimally coarsened resolution for radar radial-velocity data compression with zero or near-zero minimal information loss.

As mentioned at the end of the previous subsection, the simplified $B(r)$ in (17b) can be used as an intermediate approximation of the original background radial-velocity error covariance $B(\mathbf{r})$ in $(17 \mathrm{a})$ to compute the information losses caused by radial-velocity super-observations. This is indeed the case as shown in this subsection. In addition to this proxy use, $B(r)$ can be also used directly to model the pure-scalar error covariance without approximation for the background reflectivity field if $\sigma^{2}$ in (17b) is viewed as the background reflectivity error variance. In this case, the radial-velocity observations in Fig. 5a and background field in Fig. 5b can be viewed as reflectivity observations and background field, respectively. Then, the SVD-computed information losses with $\mathbf{B}$ constructed from $B(r)$ become accurate results (without approximation) for reflectivity super-observations, and their close comparisons with the spectral-estimated information losses (shown by the DILS1b and DIL-Spc curves in Fig. 6) ought to indicate that the spectral formulations can have the same (or even better) utility for radar reflectivity observations as demonstrated for the radar radial-velocity observations in this section.

\section{Conclusions}

In this paper, the spectral formulations derived in X11 for measuring information contents from uniformly distributed observations are applied to radar observations to test and demonstrate the advantages of the spectral formulations in comparison with the singular-value formulations derived in X07 and to explore the utilities of the spectral formulations for radar data compression. The main results are summarized below with conclusions:

(i) For uniformly distributed observations, the spectral formulations are shown to have precise correspondences to the singular-value formulations, as speculated in the conclusion of $\mathrm{X} 11$. As the SVD of the scaled observation operator $\mathbf{M}$ is generalized to complex domain in the spectral formulations, the absolute singular values can be computed very efficiently or even derived analytically in the spectral space [see Fig. 1 and (11)]. In this case, the spectral formulations are not only very accurate but also much more efficient than the singular-value formulations in computing the information contents. This advantage and related utilities of the spectral formulations for computing the information losses (if any) caused by uniform thinning, spectral truncation and local averaging are demonstrated (in Sections 2.2-2.5) with one-dimensional radar radial-velocity observations uniformly distributed along the radar beam.

(ii) For densely but non-uniformly distributed observations, the spectral formulations are shown to be able to estimate the dispersion part of the information content (measured globally by the dispersion part of relative entropy $D s$ or the Shannon entropy difference $S D$ ) with the observation error power spectrum constructed approximately based on the averaged observation resolution in each direction [see (19)]. The spectral formulations can also estimate the dispersion part of the information content from super-observations generated by local averaging with the super-observation error power spectrum constructed based on the super-observation resolution in each direction [see (20)]. These utilities of the spectral formulations are demonstrated with two-dimensional radar radial-velocity observations densely but non-uniformly distributed on the conical surface of radar scan (see Fig. 5a). The spectral-estimated information contents are closely verified by the SVD-computed counterpart values (see Table 1).

(iii) The background error power spectrum can be derived analytically [see (18)] in addition to the observation and superobservation error power spectra, so the information content (measured globally by $D s$ or $S D$ ) from the original observations and the information loss caused by their compressed superobservations through local averaging can be estimated very efficiently by the spectral formulations. The high-computational efficiency is an important advantage for the spectral formulations over the singular-value formulations, especially when the background and observation spaces are both extremely large and too large to be computed by the singular-value formulations, as often seen in radar data assimilation (Gao et al., 2004; Xu et al. 2010). Thus, regardless of the sizes of the observation and background spaces, the spectral formulations can always efficiently estimate the information loss caused by local averaging as a function of successively coarsened super-observation resolution, so an optimally coarsened super-observation resolution can be found for 
radar data compression to minimize the information loss to an insignificant level. This utility is demonstrated with the aforementioned two-dimensional radar radial-velocity observations in section 3.3 (see Fig. 6).

In addition to the results summarized above, the spectral formulations can also estimate the dispersion part of information content from thinned non-uniform observations and the associated information loss (not shown), while the thinned observation error power spectrum can be constructed based on the thinned observation resolution in each direction similarly to that for the original observations [see (19)]. Moreover, as explained at the end of Section 3.3, the original non-isotropic background radial-velocity error covariance [in (17a)] is simplified into an isotropic form [in (17b)] to facilitate the use of the spectral formulations and such an isotropic form can be used directly to model the pure-scalar error covariance without approximation for the background reflectivity field (or any other pure-scalar background field). Thus, although the spectral formulations are tested only with the radial-velocity observations, they can be equally well or even better tested with radar reflectivity observations for similar utilities. The spectral formulations are also expected to have similar utilities for other densely and remotely sensed observations (such as those from GOES satellites).

As explained in Sections 2.1 and 3.1, the original radar observations are thinned to facilitate the tests in Sections 2.5 and 3.3 for super-Obbing by local averaging to cause zero or minimal information loss. In addition, the thinning also reduced the computational cost of the singular-value formulations drastically (especially if the number of observations exceeds the order of $10^{3}$ ) for the two-dimensional case tested in Section 3 . Ideally for real-data applications (without using the expensive singular-value formulations for verifications), the spectral formulations should be applied to the original radar observations directly without thinning to avoid thinning-caused information loss (see section 4.2 of X11 and Section 2.3 of this paper). In this case, we need to consider correlated radar observation errors between neighbouring gates or neighbouring beams (Xu et al., 2007) and use their associated non-constant observation error power spectrum [more complex than that in (19)] in the spectral formulations to estimate the information contents from the original observations and evaluate the information loss caused by local averaging. This problem will be examined in a follow-up study.

\section{Acknowledgments}

The authors are thankful to Dr. Jidong Gao and Dr. David Parrish for their comments and suggestions that improved the presentation of the results. The research work was supported by the ONR Grant N000141010778 to CIMMS, the University of Oklahoma. Funding was also provided by NOAA/Office of Oceanic and Atmospheric Research under NOAA-University of Okla- homa Cooperative Agreement \#NA17RJ1227, U.S. Department of Commerce.

\section{References}

Alpert, J. and Kumar, K. 2007. Radial wind super-obs from the WSR88D radars in the NCEP operational assimilation system. Mon. Wea. Rev. 135, 1090-1109.

Frehlich, R. 2008. Atmospheric turbulence component of the innovation error covariance. Quart. J. Roy. Meteor. Soc. 134, 931-940.

Gao, J., Xue, M., Brewster, K. and Droegemeier, K. K. 2004. A threedimensional variational data assimilation method with recursive filter for single-Doppler radar. J. Atmos. Oceanic. Technol. 21, 457-469.

Golub, G. H. and Van Loan, C. F. 1983. Matrix Computations. Johns Hopkins University Press, Baltimore, 476 pp.

Hodur, R. M. 1997. The Naval Research Laboratory's coupled ocean/atmosphere mesoscale prediction system (COAMPS). Mon. Wea. Rev. 125, 1414-1430.

Lighthill, M. J. 1958. Introduction to Fourier Analysis and Generalized Functions. Cambridge University Press, New York, 79 pp.

Liu, S., Xue, M., Gao, J. and Parrish, D. 2005. Analysis and impact of super-obbed Doppler radial velocity in the NCEP grid-point statistical interpolation (GSI) analysis system. In: Proceedings of the 17th Conference on Numerical Weather Prediction. American Meteorological Society, Washington DC, 13A.4.

Lu, H., Xu, Q., Yao, M. and Gao, S. 2011. Time-Expanded sampling for ensemble-based filters: assimilation experiments with real radar observations. Adv. Atmos. Sci. 28, doi:10.1007/s00376-010-0021-4.

Purser, R. J., Parrish, D. F. and Masutani, M. 2000. Meteorological observational data compression; An alternative to conventional "super-Obbing". Office Note 430, National Centers for Environmental Prediction, Camp Springs, MD, 12 pp. Available at: http://www.emc.ncep.noaa.gov/officenotes/FullTOC.html\#2000 (last accessed 20 Apr 2011).

$\mathrm{Xu}, \mathrm{Q} .2007$. Measuring information content from observations for data assimilation: relative entropy versus Shannon entropy difference. Tellus 59A, 198-209.

Xu, Q. 2011. Measuring information content from observations for data assimilation: spectral formulations and their implications to observational data compression. Tellus 63A, 793-804.

$\mathrm{Xu}$, Q. and Gong, J. 2003. Background error covariance functions for Doppler radial-wind analysis. Quart. J. Roy. Meteor. Soc. 129, 1703-1720.

Xu, Q., Nai, K. and Wei, L. 2007. An innovation method for estimating radar radial-velocity observation error and background wind error covariances. Quart. J. Roy. Meteor. Soc. 133, 407-415.

Xu, Q., Nai, K., Wei, L., Zhang, P., Wang, L., and co-authors. 2005. Progress in Doppler radar data assimilation. In: Proceedings of the 32nd Conference on Radar Meteorology, American Meteorological Society., Albuquerque, New Mexico, JP1J7.

Xu, Q., Wei, L., Gu, W., Gong, J. and Zhao, Q. 2010. A 3.5-dimensional variational method for Doppler radar data assimilation and its application to phased-array radar observations. Adv. Meteorol. 2010, 14, doi:10.1155/2010/797265.

Xu, Q., Wei, L. and Healy, S. 2009. Measuring information content from observations for data assimilations: connection between different measures and application to radar scan design. Tellus 61A, 144-153. 